\title{
Fukaya Categories as Categorical Morse Homology ${ }^{\star}$
}

\author{
David NADLER
}

Department of Mathematics, University of California, Berkeley, Berkeley, CA 94720-3840, USA

E-mail: nadler@math.berkeley.edu

URL: http://math.berkeley.edu/ nadler/

Received May 16, 2012, in final form February 21, 2014; Published online March 01, 2014

http://dx.doi.org/10.3842/SIGMA.2014.018

\begin{abstract}
The Fukaya category of a Weinstein manifold is an intricate symplectic invariant of high interest in mirror symmetry and geometric representation theory. This paper informally sketches how, in analogy with Morse homology, the Fukaya category might result from gluing together Fukaya categories of Weinstein cells. This can be formalized by a recollement pattern for Lagrangian branes parallel to that for constructible sheaves. Assuming this structure, we exhibit the Fukaya category as the global sections of a sheaf on the conic topology of the Weinstein manifold. This can be viewed as a symplectic analogue of the well-known algebraic and topological theories of (micro)localization.
\end{abstract}

Key words: Fukaya category; microlocalization

2010 Mathematics Subject Classification: 53D37

\section{Introduction}

To realize "compact, smooth" global objects as glued together from simpler local pieces, one often pays the price that the local pieces are "noncompact" or "singular". For several representative examples, one could think about compact manifolds versus cells and simplices, smooth projective varieties versus smooth affine varieties and singular hyperplane sections, vector bundles with flat connection versus regular holonomic $\mathcal{D}$-modules, or perhaps most universally of all, irreducible modules versus induced modules.

In this paper, we informally sketch how a similar pattern might hold for exact Lagrangian branes in Weinstein manifolds: it should be possible to systematically glue together (in the homological sense) complicated branes from simple but noncompact branes. If the initial branes are compact, then Seidel's theory of iterated Lefschetz fibrations and their vanishing cycles and thimbles [55] provides a complete solution, both theoretically and computationally. For applications to some questions in geometric representation theory, it is useful to go further and consider noncompact branes from the start. When the Weinstein manifold is a cotangent bundle, constructible sheaves capture the structure of all branes, compact and noncompact alike, and in particular, organize the gluing relations between local and global calculations [41, 42, 43]. We turn here to a basic structure of a Weinstein manifold and discuss decomposing branes along its unstable coisotropic Morse cells. One can interpret the resulting proposed presentation of the Fukaya category as a categorical form of Morse homology.

As a consequence, assuming such a gluing pattern, we derive a canonical localization of branes: we construct a sheaf of categories whose global sections recover the Fukaya category. This provides a symplectic counterpart to the algebraic theory of Beilinson-Bernstein localization [8] and the topological microlocalization of Kashiwara-Schapira [27]. Though we are exclusively occupied here with general structure, we anticipate applications of potentially broad appeal. The localization of branes should assist in the calculation of many Fukaya categories, in particular

\footnotetext{
*This paper is a contribution to the Special Issue "Mirror Symmetry and Related Topics". The full collection is available at http://www.emis.de/journals/SIGMA/mirror_symmetry.html
} 
in terms of algebraic quantizations when the Weinstein manifold is the Hamiltonian reduction of a cotangent bundle. For the simplest example, one could consider Slodowy slices and their smoothings: on the one hand, their compact branes have been studied extensively in the context of knot homologies [28,37,60]; on the other hand, their noncommutative modules globalize to modules over Lie algebras and finite $W$-algebras (see [30, 32, 33, 48] for origins and up-to-date lists of references). More broadly, one could also consider other symplectic settings for geometric representation theory, for example for localizations of Cherednik-type algebras and hypertoric enveloping algebras [16, 23, 24, 26, 40]. This paper suggests useful tools to see that such targets admit a single categorical quantization which can be described in terms of localized branes or alternatively noncommutative modules.

From a concrete geometric perspective, we have been particularly inspired by Seidel's theory of exact symplectic Dehn twists [51, 52, 53, 55]. For our understanding of the foundations of the subject, we have turned to the work of Eliashberg, Gromov, and Weinstein [19, 20, 75], Fukaya, Oh, Ohta, and Ono [22], Seidel [55], Fukaya and Oh [21], Wehrheim and Woodward [67, 68, 69, 70, 71, 72], Kashiwara and Schapira [27] and Lurie [34, 35, 36]. There is a wealth of results in closely aligned directions, in particular in the analysis of the homology of closed strings. We are familiar with only a small part of this growing literature, in particular, the guiding advances of Seidel [54,56], and the striking results of Bourgeois, Cieliebak, Eckholm, and Eliashberg [14, 15, 17].

This paper outlines anticipated structure and does not provide comprehensive arguments. Due to the high technical demands, our discussion of functors on noncompact branes is only a sketch highlighting our main expectations within this rapidly developing subject. Assuming these structural results, we provide complete arguments for the localization of the Fukaya category.

In what follows, we first outline the specific setup adopted in this paper, then go on to describe our main expectations, results and their immediate precursors.

\subsection{Setup}

Let $(M, \theta)$ be a Weinstein manifold, so a manifold $M$ equipped with a one-form $\theta$ whose differential $\omega=d \theta$ is a symplectic form such that there exists a suitably compatible Morse function $h: M \rightarrow \mathbb{R}$. (We refer the reader to Section 2 below for more details on the compatibility, but note here the potential point of confusion: we prefer the flexibility of not including the choice of a particular Morse function in the data, though it is standard to do so in some other contexts.) We will always assume that $M$ is real analytic, and all subsets and functions are subanalytic (or definable within some fixed o-minimal context). The basic source of examples are Stein manifolds, or more specifically, smooth affine complex varieties.

Let $Z$ be the Liouville vector field on $M$ characterized by $\theta=i_{Z} \omega$, and let $\mathfrak{c} \subset M$ be the finite subset of zeros of $Z$. For generic data, the flow of $Z$ provides a stratification $\mathcal{S}=\left\{C_{p}\right\}_{p \in \mathfrak{c}}$ into coisotropic unstable cells $C_{p} \subset M$ contracting to the zeros $p \in \mathfrak{c}$. Hamiltonian reduction along each coisotropic unstable cell $C_{p} \subset M$ produces a contractible Weinstein manifold $\left(M_{p}, \theta_{p}\right)$ which we refer to as a Weinstein cell.

Let $F(M)$ denote the Fukaya category of not necessarily compact exact Lagrangian branes, and let Perf $F(M)$ denote the stable Fukaya category of perfect modules over $F(M)$. Here we fix a coefficient field $k$, and by perfect modules, mean summands of finite complexes of representable modules with values in $k$-chain complexes. Let us briefly orient the reader as to what version of the Fukaya category we work with. (See Section 3 below for a more detailed discussion of all of the following notions.)

First, recall that any symplectic manifold $M$ is canonically oriented, and one can unambiguously speak about its Chern classes. To work with graded Lagrangian submanifolds, we will 
make the standard assumption that the characteristic class $2 c_{1}(M)$ is trivialized. For simplicity, we will also assume that $M$ comes equipped with a spin structure. Then by Lagrangian brane, we will mean a graded Lagrangian submanifold equipped with a finite-dimensional local system and pin structure.

Second, by the Fukaya category $F(M)$, we mean the infinitesimal (as describes the setting of $[41,43,52,55]$ ) rather than wrapped variant (as found in [4]; this is one extreme of the partially wrapped paradigm $[6,7]$ ). Our perturbation framework involves Hamiltonian isotopies (in the direction of the rotated Liouville vector field) of constant size with respect to a radial coordinate near infinity rather than of linear growth. If one specifies a conic Lagrangian support $\Lambda \subset M$ for branes, there is a resulting full subcategory $\operatorname{Perf}_{\Lambda} F(M) \subset \operatorname{Perf} F(M)$. The corresponding partially wrapped category might be viewed as its "Verdier dual" (the relation appears analogous to that of cohomology and homology, or more immediately, perfect and coherent $\mathcal{O}$-modules). In fact, one might expect the partially wrapped category to embed into the category of modules over $\operatorname{Perf}_{\Lambda} F(M)$. Thus by coupling results for the infinitesimal category with notions of support and homological bounds, one might expect to obtain parallel results for the partially wrapped category. We have included some further discussion of expectations in Remark 1.8 below.

\subsection{Recollement for branes}

Our first goal is to outline how the stable Fukaya category Perf $F(M)$ might be recoverable from the stable Fukaya categories Perf $F\left(M_{p}\right)$ of its Weinstein cells together with gluing data in the form of natural adjunctions.

Given a closed coisotropic submanifold $i: C \rightarrow M$ that is a union of unstable cells, we will sketch an expected semiorthogonal decomposition of Perf $F(M)$. On the one hand, the formalism of Hamiltonian reduction provides a Lagrangian correspondence

$$
N \ll \stackrel{q}{\ll} C \stackrel{i}{\longrightarrow} M,
$$

where $N$ is a Weinstein manifold, and $q$ is the quotient along the integrable isotropic foliation determined by $i$. On the other hand (as explained in Lemma 2.25 below), the open complement $j: M^{\circ}=M \backslash C \rightarrow M$ naturally inherits the structure of a Weinstein manifold. (Note that we do not include a specific compatible Morse function in the data of a Weinstein manifold. The compatible Morse function we construct for $M^{\circ}$ is not simply the restriction of a compatible Morse function for $M$.)

We will outline how there might be a recollement pattern for Lagrangian branes analogous to standard gluings for constructible sheaves (as recalled in Section 1.4.2 below): it is a natural diagram of adjunctions

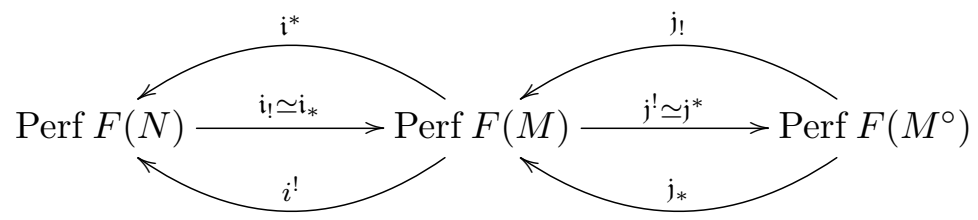

with $\mathfrak{i}_{!} \simeq \mathfrak{i}_{*}, \mathfrak{j}_{!}, \mathfrak{j}_{*}$ fully faithful embeddings, along with exact triangles of functors

$$
\mathfrak{i}_{i} \mathfrak{i}^{!} \stackrel{c}{\longrightarrow} \operatorname{idd}_{\operatorname{Perf} F(M)} \stackrel{u}{\longrightarrow} \mathfrak{j}_{*} \mathfrak{j}^{*} \stackrel{[1]}{\longrightarrow}, \quad \mathfrak{j}_{!} \mathfrak{j}^{!} \stackrel{c}{\longrightarrow} \operatorname{id} \operatorname{Perf} F(M) \stackrel{u}{\longrightarrow} \mathfrak{i}_{*} \mathfrak{i}^{*} \stackrel{[1]}{\longrightarrow},
$$

where $c$ denotes the counits of adjunctions and $u$ the units.

From a symplectic perspective, the functors involved are "Dehn twists around cells". Alternatively, one can also interpret them as resulting from Lagrangian surgery. 
Remark 1.1. If $M$ admits an anti-symplectomorphism compatible with other structures, then one can obtain an analogue of Verdier duality as well.

Remark 1.2. The exact triangles are categorical versions of the long exact sequences of cohomology of a pair. It is useful to observe that they are formally equivalent to exact triangles

$$
\operatorname{id}_{F(M)} \longrightarrow \mathfrak{j}_{*} \mathfrak{j}^{*} \oplus \mathfrak{i}_{*} \mathfrak{i}^{*} \longrightarrow \mathfrak{i}_{*} \mathfrak{i}^{*} \mathfrak{j}_{*} \mathfrak{j}^{*} \stackrel{[1]}{\longrightarrow}, \quad \mathfrak{i}_{!} i^{\prime} \mathfrak{j}_{\mathfrak{j}} \mathfrak{j}^{!} \longrightarrow \mathfrak{j}_{!} \mathfrak{j}^{!} \oplus \mathfrak{i}_{!} i^{!} \longrightarrow \operatorname{id}_{F(M)} \stackrel{[1]}{\longrightarrow},
$$

where the first and second maps are the units and counits of adjunctions. These are categorical versions of the Mayer-Vietoris sequences of cohomology of a covering pair.

Remark 1.3. We mention here an attractive way to potentially repackage the recollement pattern.

Consider the differential graded derived category $\operatorname{Sh}_{\mathcal{S}}(M)$ of complexes of sheaves constructible along the stratification $\mathcal{S}=\left\{C_{p}\right\}_{p \in \mathfrak{c}}$ by coisotropic unstable cells $C_{p} \subset M$. We equip $\mathrm{Sh}_{\mathcal{S}}(M)$ with its natural symmetric monoidal structure given by tensor product. The monoidal unit is the constant sheaf $k_{M} \in \mathrm{Sh}_{\mathcal{S}}(M)$. More generally, given a union of unstable coisotropic cells $i: C \rightarrow M$, the extension by zero $k_{C !}=i_{!} k_{C} \in \operatorname{Sh}_{\mathcal{S}}(M)$ is an idempotent.

Now given the recollement functors, it seems likely one should be able to reorganize them into a natural fully faithful monoidal embedding

$$
\mathrm{Sh}_{\mathcal{S}}(M) \longrightarrow \operatorname{End}_{\mathbf{s t}_{k}}(\operatorname{Perf} F(M))
$$

characterized by the property that $k_{C !} \longmapsto \mathfrak{i}_{1} i^{*}$. This would allow one to analyze Perf $F(M)$ as a module over the "spectrum" of the commutative algebra $\mathrm{Sh}_{\mathcal{S}}(M)$.

Such a recollement pattern would provide various frameworks for gluing together Perf $F(M)$ from the constituent pieces Perf $F(N)$, Perf $F\left(M^{\circ}\right)$. To pursue gluing, it is useful to regard each of the above categories as a small stable idempotent-complete $k$-linear $\infty$-category. Then we can adopt the foundations of [34,35], and work within the $\infty$-category st $_{k}$ of small stable idempotent-complete $k$-linear $\infty$-categories.

According to the reinterpretation of Remark 1.2, we can view Perf $F(M)$ as classifying triples of data $L^{\circ} \in \operatorname{Perf} F\left(M^{\circ}\right), L_{N} \in \operatorname{Perf} F(N)$ together with a morphism

$$
r \in \operatorname{Hom}_{\operatorname{Perf} F(N)}\left(\mathfrak{i}^{!} \mathfrak{j}_{!} L^{\circ}, L_{N}\right) .
$$

Let us recast this in monadic terms by considering the adjunction

$$
L=\mathfrak{j}_{!} \oplus \mathfrak{i}_{!}: \operatorname{Perf} F\left(M^{\circ}\right) \oplus \operatorname{Perf} F(N) \longleftrightarrow \operatorname{Perf} F(M): R=\mathfrak{j}^{!} \oplus \mathfrak{i}^{!} .
$$

We obtain a resulting monad, or in other words, algebra object in endomorphisms

$$
T=R L \in \operatorname{End}\left(\operatorname{Perf} F\left(M^{\circ}\right) \oplus \operatorname{Perf} F(N)\right) .
$$

The $\infty$-categorical Barr-Beck theorem provides a canonical equivalence

$$
\operatorname{Perf} F(M) \simeq \operatorname{Mod}_{T}\left(\operatorname{Perf} F\left(M^{\circ}\right) \oplus \operatorname{Perf} F(N)\right),
$$

where the right hand side denotes module objects over the monad $T$.

We can inductively apply the above considerations by successively taking the coisotropic submanifold $i: C \rightarrow M$ to be a single closed unstable cell. Recall that $\mathfrak{c} \subset M$ denotes the finite subset of zeros of the Liouville vector field $Z$. For each $p \in \mathfrak{c}$, we have the Hamiltonian reduction of the corresponding unstable cell

$$
M_{p} \stackrel{q_{p}}{\longleftarrow} C_{p} \stackrel{i_{p}}{\longrightarrow} M
$$


By induction, the recollement pattern provides an adjunction

$$
L=\oplus_{p \in \mathfrak{c}} \mathfrak{i}_{p !}: \oplus_{p \in \mathfrak{c}} \operatorname{Perf} F\left(M_{p}\right) \longleftrightarrow \operatorname{Perf} F(M): R=\oplus_{p \in \mathfrak{c}} \mathfrak{i}_{p}^{!} .
$$

We obtain a resulting upper-triangular monad

$$
T=R L \in \text { End }\left(\oplus_{p \in \mathfrak{c}} \operatorname{Perf} F\left(M_{p}\right)\right)
$$

with matrix entries $\mathfrak{i}_{q}^{!} \mathfrak{i}_{p}$ ! for unstable cells $C_{q} \subset \bar{C}_{p}$. Finally, the $\infty$-categorical Barr-Beck theorem provides a canonical equivalence

$$
\operatorname{Perf} F(M) \simeq \operatorname{Mod}_{T}\left(\oplus_{p \in \mathfrak{c}} \operatorname{Perf} F\left(M_{p}\right)\right)
$$

One can view this presentation of Perf $F(M)$ as a categorified version of the Morse homology of $M$. First, to each critical point $p \in \mathfrak{c}$, we assign the stable Fukaya category Perf $F\left(M_{p}\right)$ of the Weinstein cell $M_{p}$. From the perspective of Morse homology, one can view $F\left(M_{p}\right)$ as the analogue of a scalar vector space $k[\operatorname{deg} p]$ shifted by the index of $p$. (Since the $\infty$-category st $k$ of small stable idempotent-complete $k$-linear $\infty$-categories is not itself stable, it is unsurprising that "suspension" in the current setting is not invertible as it is in the traditional setting.)

Second, to pairs of critical points $p, q \in \mathfrak{c}$, we assign the individual term $\mathfrak{i}_{q}^{!} \mathfrak{i}_{p}$ ! of the gluing monad $T$. More generally, to collections of critical points, we assign the corresponding terms of the gluing monad $T$ together with the monadic structure maps among them. From the perspective of Morse homology, one can view the individual terms and their monadic structure as the analogue of boundary maps and their higher relations.

Part of the appeal of Morse homology is that the boundary maps and their higher relations localize along the spaces of flow lines connecting the relevant critical points. Furthermore, the boundary maps and their higher relations admit simple descriptions in terms of the spaces of flow lines. For instance, the boundary maps themselves are given by counts of isolated flow lines. We will develop an abstract analogue of this picture directly below.

\subsection{Localization of branes}

Now let us assume the above recollement pattern and see what it further implies. We will deduce a localization of the stable Fukaya category Perf $F(M)$ over the conic topology of $M$. There are many inspiring precedents $[1,4,57,59,62]$ and informed assertions [29] that suggest such a construction should be possible. In our own thinking, we have often returned to the setting of cotangent bundles as a guide: combining the equivalence of Lagrangian branes with constructible sheaves $[41,42,43]$ and the formalism of microlocalization [27] leads to a complete solution in that case.

To describe the localization for a general Weinstein manifold $(M, \theta)$, let us return to the geometry of the Liouville vector field $Z$. We say a subset of $M$ is conic if it is invariant under the flow of $Z$, and use the term core to refer to the compact conic isotropic subvariety $K \subset M$ of points that do not escape to infinity under the flow. (Many authors prefer the term "skeleton" for $K \subset M$, but we have opted for core so as not to confuse it with the characteristic cone $\Lambda \subset M$ introduced below. There is a growing number of authors who would refer to $\Lambda \subset M$ as a noncompact skeleton, and so we prefer to distinguish the compact skeleton $K \subset M$ with the name core.) We use the term ether to refer to the complement $E=M \backslash K$, and define the projectivization $M^{\infty}=E / \mathbb{R}_{+}$to be the compact contact manifold obtained by quotienting the ether by the flow. More generally, given any conic subset $A \subset M$, we can consider its projectivization $A^{\infty}=(A \cap E) / \mathbb{R}_{+} \subset M^{\infty}$.

To any object $L \in \operatorname{Perf} F(M)$, we assign its singular support $\operatorname{ss}(L) \subset M$ which is a closed conic isotropic subvariety depending only on the isomorphism class of the object (see Section 3.7 
below). The singular support records the coarse homological nontriviality of objects, and for a Lagrangian brane is a subset of its limiting dilation to a conic Lagrangian subvariety. For example, if $L$ is a compact Lagrangian brane, then $\operatorname{ss}(L) \subset K$.

Let us fix a (most likely singular and noncompact) conic Lagrangian subvariety $\Lambda \subset M$ which we refer to as the characteristic cone. We will assume that $\Lambda$ contains the core $K \subset M$, and hence is completely determined by its projectivization $\Lambda^{\infty} \subset M^{\infty}$. Note that the inclusions $K \subset \Lambda \subset M$ are all homotopy equivalences, so what will interest us most is their local geometry.

Let us consider the full subcategory $\operatorname{Perf}_{\Lambda}(M) \subset \operatorname{Perf} F(M)$ of objects $L \in \operatorname{Perf} F(M)$ with singular support satisfying $\operatorname{ss}(L) \subset \Lambda$. So for example, if $L$ is a compact Lagrangian brane, then it provides an object of $\operatorname{Perf}_{\Lambda} F(M)$ irrespective of the choice of $\Lambda$. Note that $\Lambda_{1} \subset \Lambda_{2}$ implies $\operatorname{Perf}_{\Lambda_{1}} F(M) \subset \operatorname{Perf}_{\Lambda_{2}} F(M)$, and Perf $F(M)=\cup_{\Lambda} \operatorname{Perf}_{\Lambda} F(M)$.

Recall that $\mathfrak{c} \subset M$ denotes the finite subset of zeros of the Liouville vector field $Z$. For each $p \in \mathfrak{c}$, we have the Hamiltonian reduction of the corresponding unstable cell

$$
M_{p} \stackrel{q_{p}}{\longleftarrow} C_{p} \stackrel{i_{p}}{\longrightarrow} M
$$

We obtain a conic Lagrangian subvariety $\Lambda_{p} \subset M_{p}$ by setting $\Lambda_{p}=q_{p}\left(i_{p}^{-1}(\Lambda)\right)$, and similarly a full subcategory $\operatorname{Perf}_{\Lambda_{p}} F\left(M_{p}\right) \subset \operatorname{Perf} F\left(M_{p}\right)$.

Now by localizing $\operatorname{Perf}_{\Lambda} F(M)$ with respect to singular support, we obtain the following. Its verification appeals to the recollement pattern outlined above.

Theorem 1.4. Assume the recollement pattern of diagrams (1.1) and (1.2).

There exists a $\mathrm{st}_{k}$-valued sheaf $\mathcal{F}_{\Lambda}$ on the conic topology of $M$ with the following properties:

(1) The support of $\mathcal{F}_{\Lambda}$ is the characteristic cone $\Lambda \subset M$.

(2) The global sections of $\mathcal{F}_{\Lambda}$ are canonically equivalent to $\operatorname{Perf}_{\Lambda} F(M)$.

(3) The restriction of $\mathcal{F}_{\Lambda}$ to an open Weinstein submanifold $M^{\circ} \subset M$ is canonically equivalent to the sheaf $\mathcal{F}_{\Lambda^{\circ}}$ constructed with respect to $\Lambda^{\circ}=\Lambda \cap M^{\circ}$.

(4) For each zero $p \in \mathfrak{c}$, the sections of $\mathcal{F}_{\Lambda}$ lying strictly above the unstable cell $C_{p} \subset M$ are canonically equivalent to $\operatorname{Perf}_{\Lambda_{p}} F\left(M_{p}\right)$.

Example 1.5. Here is a description of the sheaf $\mathcal{F}_{\Lambda}$ in the simplest example.

Consider the two-dimensional Weinstein cell $M=\mathbb{C}$ with standard Liouville form $\theta$ and projectivization $M^{\infty} \simeq S^{1}$. Its core is the single point $K=\{0\} \subset \mathbb{C}$, and its ether is the complement $E=\mathbb{C}^{*} \subset \mathbb{C}$. Any characteristic cone $\Lambda \subset \mathbb{C}$ will be the union of $K=\{0\}$ with finitely many rays. For $n=0,1,2, \ldots$, let $\Lambda_{n} \subset \mathbb{C}$ denote the characteristic cone with $n$ rays.

Then for $n>1, \operatorname{Perf}_{\Lambda_{n}} F(M)$ is equivalent to finite-dimensional modules over the $A_{n-1^{-}}$ quiver $1 \rightarrow 2 \rightarrow \cdots \rightarrow n$. For $n=0$ or 1 , it is the zero category. This is also the stalk of the sheaf $\mathcal{F}_{\Lambda_{n}}$ at the point $0 \in \mathbb{C}$. Its stalk at other points $x \in \mathbb{C}$ is (not necessarily canonically) equivalent to Perf $k$ when $x \in \Lambda_{n}$, and is the zero category otherwise.

Example 1.6. As a continuation of the previous example (or in fact a direct generalization), we could take $M$ to be an open Riemann surface of genus $g$ with $k>0$ punctures. Then its projectivization $M^{\infty}$ is the disjoint union of $k$ circles, and its core $K \subset M$ is a graph homotopy equivalent to a bouquet of $2 g+(k-1)$ circles. Any characteristic cone $\Lambda \subset M$ will be the union of $K$ with finitely many rays shooting from the nodes of $K$ off to the boundary. So all together, we can view $\Lambda$ as a "ribbon graph": an abstract graph with some noncompact edges embedded in the Riemann surface $M$ as a retract.

Now the sheaf $\mathcal{F}_{\Lambda}$ is supported along $\Lambda$ (so equivalently can be viewed as a sheaf on $\Lambda$ ). It is locally constant along the edges of $\Lambda$ with stalks (not necessarily canonically) equivalent to Perf $k$. At the nodes of $\Lambda$, it is (not necessarily canonically) equivalent to finite-dimensional 
modules over the $A_{n}$-quiver where $n+2$ is the valency of a node. (So if a node has valency 0 or 1 , the stalk is the zero category.) The global sections $\operatorname{Perf}_{\Lambda}(M)$ can be easily calculated as the global sections of $\mathcal{F}_{\Lambda}$, with the combinatorial form of the answer dependent on the presentation of the data of $\Lambda$ and its embedding in $M$. (For example, this is compatible with the "constructible plumbing model" for ribbon graphs with valency $\leq 4$ developed in [62].)

Remark 1.7. It is possible to say much more about $\mathcal{F}_{\Lambda}$. Let us content ourselves here with mentioning that when $M$ is a cotangent bundle, sections of $\mathcal{F}_{\Lambda}$ are equivalent to (the sheafification of) microlocal sheaves. In general, a similar description holds for the restriction of $\mathcal{F}_{\Lambda}$ to the complement $M \backslash \mathfrak{c}$ of the zeros of the Liouville vector field $Z$. Namely, the quotient $(M \backslash \mathfrak{c}) / \mathbb{R}_{+}$ by the Liouville flow is naturally a (non-Hausdorff) contact manifold, and the restriction of $\mathcal{F}_{\Lambda}$ to the complement $M \backslash \mathfrak{c}$ can be obtained by pulling back microlocal sheaves from $(M \backslash \mathfrak{c}) / \mathbb{R}_{+}$.

Remark 1.8. This remark is devoted to a conjectural parallel picture for the partially wrapped Fukaya category. Let us continue with the setting of Theorem 1.4, and write $W F_{\Lambda}(M)$ for the partially wrapped Fukaya category as developed in [6, 7]. It specializes to the fully wrapped variant of [4] when the characteristic cone $\Lambda \subset M$ coincides with the compact core $K \subset M$.

Let Perf $W F_{\Lambda}(M)$ denote the stable category of perfect modules over $W F_{\Lambda}(M)$, and let Mod $W F_{\Lambda}(M)$ denote the stable category of all modules. Note that the two stabilizations are formally the same amount of information: $\operatorname{Mod} W F_{\Lambda}(M)$ is the ind-category $\operatorname{Ind}\left(\operatorname{Perf} W F_{\Lambda}(M)\right)$, and Perf $W F_{\Lambda}(M)$ is the full subcategory of compact objects $\left(\operatorname{Mod} W F_{\Lambda}(M)\right)^{c}$.

Given a conic open subset $U \subset M$, let us imagine a "mirror" picture of the category $\mathcal{F}_{\Lambda}(U)$ as perfect quasicoherent sheaves over a scheme $X_{U}$ proper over Spec $k$. The statement that $X_{U}$ is proper can be formalized by the expectation that $\mathcal{F}_{\Lambda}(U)$ is hom-finite. Given conic open subsets $V \subset U \subset M$, let us also imagine a "mirror" picture of the restriction map $\mathcal{F}_{\Lambda}(U) \rightarrow \mathcal{F}_{\Lambda}(V)$ as the pullback of perfect quasicoherent sheaves under a morphism $X_{V} \rightarrow X_{U}$.

Now given a conic open subset $U \subset M$, consider the category of finite functionals

$$
\operatorname{Coh} \mathcal{F}_{\Lambda}(U)=\operatorname{Hom}_{\text {st }_{k}}\left(\mathcal{F}_{\Lambda}(U)^{\mathrm{op}}, \operatorname{Perf} k\right) .
$$

From the above "mirror" perspective, $\operatorname{Coh} \mathcal{F}_{\Lambda}(U)$ corresponds to coherent sheaves over the proper scheme $X_{U}$. Given conic open subsets $V \subset U \subset M$, the restriction map $\mathcal{F}_{\Lambda}(U) \rightarrow \mathcal{F}_{\Lambda}(V)$ induces a corestriction map

$$
\operatorname{Coh} \mathcal{F}_{\Lambda}(V) \longrightarrow \operatorname{Coh} \mathcal{F}_{\Lambda}(U) .
$$

From the above "mirror" perspective, the corestriction map corresponds to the pushforward (right adjoint to pullback) of coherent sheaves under the morphism $X_{U} \rightarrow X_{V}$.

The above constructions equip $\operatorname{Coh} \mathcal{F}_{\Lambda}$ with the structure of a pre-cosheaf. Let us write $\operatorname{Ind}\left(\operatorname{Coh} \mathcal{F}_{\Lambda}\right)$ for the corresponding pre-cosheaf of ind-categories, and $\operatorname{Ind}\left(\operatorname{Coh} \mathcal{F}_{\Lambda}\right)^{+}$for its cosheafification. (If one prefers the intuitions of sheaves over cosheaves, one could pass to the right adjoints of the corestriction maps of $\operatorname{Ind}\left(\operatorname{Coh} \mathcal{F}_{\Lambda}\right)^{+}$and turn it into a sheaf.) We expect that there is a natural equivalence

$$
\Gamma\left(M, \operatorname{Ind}\left(\operatorname{Coh} \mathcal{F}_{\Lambda}\right)^{+}\right) \stackrel{\sim}{\longrightarrow} \operatorname{Mod} W F_{\Lambda}(M) .
$$

In other words, the stable partially wrapped Fukaya category Perf $W F_{\Lambda}(M)$ consists of the compact objects of the global sections of the cosheaf $\operatorname{Ind}\left(\operatorname{Coh} \mathcal{F}_{\Lambda}\right)^{+}$.

Finally, let us mention some corroborating evidence for the above picture. First, using the results of $[2,41,43]$, one can check it for $M$ a cotangent bundle and $\Lambda$ the zero section. Second, recent calculations of Fukaya categories of Riemann surfaces, pairs-of pants and their generalizations $[3,58,61]$ reveal a mirror symmetry with matrix factorizations. In the situations considered, the wrapped Fukaya category corresponds to "coherent" matrix factorizations and the full subcategory of compact branes corresponds to "perfect" matrix factorizations. 
We have briefly mentioned anticipated applications of the recollement pattern and Theorem 1.4 earlier in the introduction. Let us conclude here with a useful technical application: Theorem 1.4 allows us to define the stable Fukaya category of an "inexhaustible, singular Weinstein manifold" (for example, an "open Weinstein cobordism"). Namely, given a union of coisotropic cells $C \subset M$, we can take the sections of the sheaf $\mathcal{F}_{\Lambda}$ lying strictly above $C$ for increasing $\Lambda$. Rather than leaving it in such an abstract form, let us explain what results by appealing directly to the concrete geometry of the recollement pattern.

To begin, let us rotate our viewpoint on the exact triangles appearing in diagram (1.2) and switch the roles of the known and unknown categories in our discussion. Recall that we assumed that the closed coisotropic subvariety $i: C \rightarrow M$ is both a smooth submanifold and a union of unstable cells. Let us relax these two requirements in turn.

First, suppose the closed coisotropic subvariety $i: C \rightarrow M$ is no longer necessarily smooth, but still a union of unstable cells. On the one hand, the formalism of Hamiltonian reduction still provides a correspondence

$$
N \stackrel{q}{\longleftarrow} C \stackrel{i}{\longrightarrow} M
$$

though $N$ is now a "singular Weinstein manifold", whatever that might mean. On the other hand, the open complement $j: M^{\circ}=M \backslash C \rightarrow M$ continues to inherit the structure of a Weinstein manifold. With this setup, the constructions underlying diagram (1.1) should provide adjunctions

$$
\mathfrak{j}^{*}: \operatorname{Perf} F(M) \rightleftarrows \operatorname{Perf} F\left(M^{\circ}\right): \mathfrak{j}_{*}, \quad \mathfrak{j}_{!}: \operatorname{Perf} F\left(M^{\circ}\right) \rightleftarrows \operatorname{Perf} F(M): \mathfrak{j}^{!}
$$

with $\mathfrak{j}_{!}, \mathfrak{j}_{*}$ fully faithful, and $\mathfrak{j}^{!} \simeq \mathfrak{j}^{*}$. We do not have an a priori definition of a Fukaya category Perf $F(N)$, but the recollement pattern tells us what it should be. Namely, we should define $F(N)$ to classify triples of data $L \in \operatorname{Perf} F(M), L^{\circ} \in \operatorname{Perf} F\left(M^{\circ}\right)$, together with a morphism $u \in \operatorname{Hom}_{\operatorname{Perf} F(M)}\left(L, \mathfrak{j}_{*} L^{\circ}\right)$. When $N$ is smooth, the recollement pattern confirms that we recover precisely Perf $F(N)$ by taking the kernel of morphisms $u$ appearing in such data.

Remark 1.9. One should view the preceding as more than a formal analogue of the situation for $\mathcal{D}$-modules on singular varieties. There Kashiwara's theorem confirms that such an approach provides an unambiguous notion of $\mathcal{D}$-module.

Second, suppose the closed coisotropic subvariety $i: C \rightarrow M$ is a smooth submanifold invariant under the Liouville flow, but not necessarily a union of unstable cells. Suppose as well that its Hamiltonian reduction $N$ is a smooth manifold, and hence a Weinstein manifold. Observe that the open complement $j: M^{\circ}=M \backslash C \rightarrow M$ may be viewed as an "inexhaustible Weinstein manifold", or informally speaking, a complete exact symplectic manifold such that the Liouville vector field is gradient-like for a possibly inexhausting Morse function. With this setup, the constructions underlying diagram (1.1) should provide adjunctions

$$
\mathfrak{i}^{*}: \operatorname{Perf} F(N) \rightleftarrows \operatorname{Perf} F(M): \mathfrak{i}_{*}, \quad \mathfrak{i}_{!}: \operatorname{Perf} F(M) \rightleftarrows \operatorname{Perf} F(N): \mathfrak{i}^{!}
$$

with $\mathfrak{i}_{!} \simeq \mathfrak{i}_{*}$ fully faithful. We do not have an a priori definition of a Fukaya category Perf $F\left(M^{\circ}\right)$, but the recollement pattern tells us what it should be. Namely, we should define Perf $F\left(M^{\circ}\right)$ to classify triples of data $L_{N} \in \operatorname{Perf} F(N), L \in \operatorname{Perf} F(M)$, together with a morphism $c \in$ Hom $_{\text {Perf } F(M)}\left(\mathfrak{i}_{!} L_{N}, L\right)$. When $C$ is a union of unstable cells, the the recollement pattern confirms that we recover precisely Perf $F\left(M^{\circ}\right)$ by taking the cokernel of morphisms $c$ appearing in such data.

Putting together the above generalizations, we obtain an unambigious infinitesimal Fukaya category of an "inexhaustible, singular Weinstein manifold" so that it is compatible with familiar notions whenever they apply. 


\subsection{Influences}

In the remainder of the introduction, we recount some influences on our thinking, in particular the symplectic geometry of Dehn twists, the recollement pattern for constructible sheaves, and the Morse theory of integral kernels.

\subsubsection{Dehn twists}

One can view our expectations as simple elaborations on the fundamental notion of exact symplectic Dehn twists. In turn, the many incarnations of this notion (spherical twists, mutations, braid actions, Hecke correspondences, ... ) play a prominent role in mirror symmetry and geometric representation theory.

We sketch here an informal picture of our expectations from the perspective of Dehn twists. The basic mantra could be: Dehn twists around spheres provide mutations; Dehn twists around cells provide semiorthogonal decompositions. (There are also extensive relations between mutations and semiorthogonal decompositions, often arising from exceptional collections, thanks to the fact that spheres themselves can be cut into cells.)

Let us recall Seidel's long exact sequence in Floer cohomology [51, 52, 53]. There are also highly relevant relative sequences found in the work of Perutz [45, 46, 47] and WehrheimWoodward [67]. We will ignore technical issues and proceed as quickly as possible to the statement.

Let $S \subset M$ be an exact Lagrangian sphere in an exact symplectic manifold. Let $\tau_{S}: M \rightarrow M$ be the associated exact symplectic Dehn twist around $S$. Then for any two exact Lagrangian submanifolds $L_{0}, L_{1} \subset M$, there is a long exact sequence of $(\mathbf{Z} / 2 \mathbf{Z}$-graded, $\mathbf{Z} / 2 \mathbf{Z}$-linear $)$ Floer cohomology groups

$$
H F\left(\tau_{S}\left(L_{0}\right), L_{1}\right) \longrightarrow H F\left(L_{0}, L_{1}\right) \longrightarrow H F\left(S, L_{1}\right) \otimes H F\left(L_{0}, S\right) \stackrel{[1]}{\longrightarrow} .
$$

The sequence admits a straightforward categorical interpretation. Assume $M$ is equipped with appropriate background structures, and the exact Lagrangian submanifolds $S, L_{0}, L_{1}$ are all equipped with appropriate brane structures. Let $\operatorname{Mod} F(M)$ denote the $\mathbf{Z}$-graded, $k$-linear stable Fukaya category of modules, and let $S, L_{0}, L_{1}$ denote the corresponding objects. Thanks to the functoriality of the sequence in the variable $L_{1}$, we can rewrite it as an exact triangle

$$
S \otimes \operatorname{Hom}_{\operatorname{Mod} F(M)}\left(S, L_{0}\right) \longrightarrow L_{0} \longrightarrow \tau_{S}\left(L_{0}\right) \stackrel{[1]}{\longrightarrow} .
$$

Let us introduce the (presently elaborate but ultimately justified) notation

$$
\mathfrak{i}_{S !}: \operatorname{Mod} F_{S}(M) \rightleftarrows \operatorname{Mod} F(M): \mathfrak{i}_{S}^{!}
$$

for the fully faithful embedding $\mathfrak{i}_{S \text { ! }}$ of the subcategory $\operatorname{Mod} F_{S}(M)$ generated by $S$, and its right adjoint $\mathfrak{i}_{S}^{!}=\operatorname{Hom}_{\operatorname{Mod} F(M)}(S,-)$. Then thanks to the functoriality of the sequence in the variable $L_{0}$, we can view it as an exact triangle of functors

$$
\mathfrak{i}_{S !} \mathfrak{i}_{S} \longrightarrow \operatorname{id}_{\operatorname{Mod} F(M)} \longrightarrow \tau_{S} \stackrel{[1]}{\longrightarrow},
$$

where the first map is the counit of the adjunction.

Now suppose the exact Lagrangian sphere $S \subset M$ were rather a closed but noncompact exact Lagrangian cell $C \subset M$. To place it in a categorical context, let us now allow the Fukaya category $F(M)$ to contain closed but noncompact Lagrangian branes. Because $C \subset M$ is now 
a cell, we will be able to work with the $\mathbf{Z}$-graded, $k$-linear stable category Perf $F(M)$ of perfect modules. Then one expects to have a similar exact triangle of functors

$$
\mathfrak{i}_{C !} i_{C}^{!} \longrightarrow \operatorname{id}_{\operatorname{Perf} F(M)} \longrightarrow \tau_{C} \stackrel{[1]}{\longrightarrow}
$$

where $\tau_{C}$ denotes the "Dehn twist" around the cell $C \subset M$. It takes compact Lagrangian branes to noncompact Lagrangian branes which are asymptotically close to $C$ near infinity.

Let us go one step further and consider the open exact symplectic submanifold $\mathfrak{j}_{M^{\circ}}: M^{\circ}=$ $M \backslash C \rightarrow M$. Assuming $C$ is in good position with respect to the exact symplectic structure, we should be able to realize $\tau_{C}$ as the monad of an adjunction

$$
\mathfrak{j}_{M^{\circ}}^{*}: \operatorname{Perf} F(M) \rightleftarrows \operatorname{Perf} F\left(M^{\circ}\right): \mathfrak{j}_{M^{\circ} *}
$$

where $j_{M^{\circ} *}$ is a fully faithful embedding. Here the functor $j_{M^{\circ} *}$ is geometric, built out of the inclusion $j_{M^{\circ}}$ and the Dehn twist $\tau_{C}$ near infinity. Its left adjoint $j_{M^{\circ}}^{*}$ is of a categorical origin, just as the left adjoint $i_{C}^{!}$is of a categorical origin.

Putting the above together, we obtain an exact triangle of functors

$$
\mathfrak{i}_{C !} \mathfrak{i}_{C}^{!} \longrightarrow \operatorname{id}_{\operatorname{Perf} F(M)} \longrightarrow \mathfrak{j}_{M^{\circ} *} \mathfrak{j}_{M^{\circ}}^{*} \stackrel{[1]}{\longrightarrow}
$$

where the initial map is the counit of the adjunction, and the middle map is the unit of the adjunction. Thus we have a semiorthogonal decomposition of $\operatorname{Perf} F(M)$ by the two full subcategories Perf $F_{C}(M)$, Perf $F\left(M^{\circ}\right)$ with the semiorthogonality Perf $F_{C}(M)^{\perp} \simeq \operatorname{Perf} F\left(M^{\circ}\right)$, Perf $F_{C}(M) \simeq{ }^{\perp} \operatorname{Perf} F\left(M^{\circ}\right)$.

We will suggest a generalization of the above picture where we allow the cell $C \subset M$ to be coisotropic rather than Lagrangian. The geometric constructions and formal consequences should be similar, with the main new development that the full subcategory $\operatorname{Perf} F_{C}(M)$ no longer should be generated by a single object. In the section immediately following, we motivate the general pattern with the formalism of recollement for constructible sheaves.

\subsubsection{Recollement pattern}

We recall here the recollement pattern for constructible sheaves.

In what follows, we will only consider subanalytic sets $X$ and subanalytic maps $f: X \rightarrow Y$. We write $\operatorname{Sh}(X)$ for the differential graded category of constructible complexes of sheaves on $X$, and have the standard adjunctions

$$
f^{*}: \operatorname{Sh}(Y) \rightleftarrows \operatorname{Sh}(X): f_{*}, \quad f_{!}: \operatorname{Sh}(X) \rightleftarrows \operatorname{Sh}(Y): f^{!} .
$$

Verdier duality provides an anti-involution

$$
\mathbb{D}_{X}: \operatorname{Sh}(X) \stackrel{\sim}{\longrightarrow} \operatorname{Sh}(X)^{\text {op }}
$$

intertwining the preceding adjunctions

$$
f^{!} \simeq \mathbb{D}_{X} f^{*} \mathbb{D}_{Y}, \quad f_{!}=\mathbb{D}_{Y} f_{*} \mathbb{D}_{X}
$$

Suppose we have a partition of $X$ into an open subset and its closed complement

$$
j: U \smile X \longleftrightarrow Y=X \backslash U: i .
$$


Then the standard functors provide a diagram

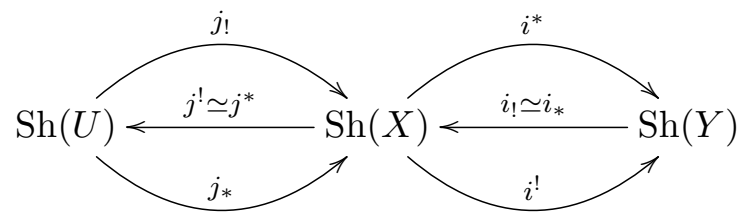

The fact that $j$ is an open embedding (hence smooth of relative dimension zero) provides a canonical identification $j^{!} \simeq j^{*}$, and the fact that $i$ is a closed embedding (hence proper) provides a canonical identification $i_{!} \simeq i_{*}$.

The fact that $U$ and $Y$ are disjoint provide canonical identifications $j^{*} i_{!} \simeq 0 \simeq \mathfrak{j}^{!} i_{*}$ and $i^{*} j_{!} \simeq 0 \simeq i^{!} j_{*}$. The additional fact that $U$ and $Y$ cover $X$ lead to dual exact triangles

$$
i_{i} i^{!} \longrightarrow \operatorname{id}_{\operatorname{Sh}(X)} \longrightarrow j_{*} j^{*} \stackrel{[1]}{\longrightarrow}, \quad j ! !^{!} \longrightarrow \operatorname{id}_{\operatorname{Sh}(X)} \longrightarrow i_{*} i^{*} \stackrel{[1]}{\longrightarrow},
$$

where the first and middle morphisms are respectively counits and units of adjunctions. These triangles are generalizations of the long exact sequences of pairs in cohomology.

Alternatively, we also have the dual exact triangles

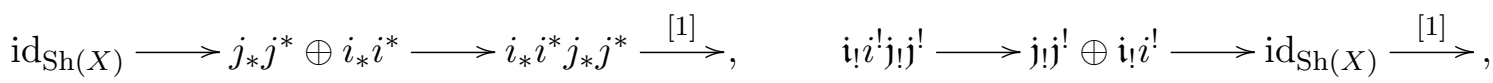

where the first and middle morphisms result from units and counits of adjunctions respectively. These triangles are generalizations of the Mayer-Vietoris long exact sequences of cohomology.

Now let us find the above formalism as a special case of the recollement pattern of diagrams (1.1) and (1.2). By inductive considerations, one can see that the above formalism devolves from the case when $X$ is a manifold and $Y \subset X$ is a submanifold. Let us focus on this case and consider the Weinstein manifolds $M=T^{*} X$ and $N=T^{*} Y$. Recall from [41, 42, 43] the microlocalization equivalence

$$
\mu_{X}: \operatorname{Sh}(X) \stackrel{\sim}{\longrightarrow} \operatorname{Perf} F\left(T^{*} X\right) .
$$

Then by an inductive sequence of applications of the recollement pattern of of diagrams (1.1) and (1.2), we obtain commutative diagrams with horizontal maps fully faithful embeddings and vertical maps equivalences
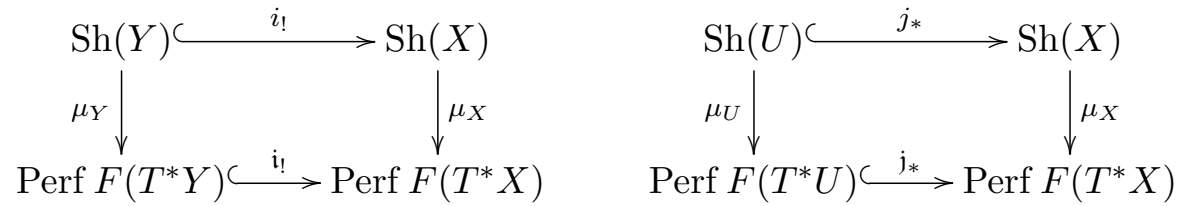

Using the natural involutive anti-symplectomorphism on a cotangent bundle, one can construct Verdier duality on its Fukaya category as well [41].

\subsubsection{Morse theory of integral kernels}

Our expectations are guided by the well-known strategy: to prove universal statements about objects of a category, one should realize endofunctors of the category as integral transforms and establish canonical identities among them.

We will illustrate this with the toy case of Morse theory since our later discussion is a direct analogue of it. For simplicity, let us work with a compact oriented manifold $M$. Consider a generic pair consisting of a Morse function $f: M \rightarrow \mathbb{R}$ and Riemannian metric $g$ on $M$. Let 
$\mathfrak{c} \subset M$ denote the critical locus of $f$, and $\Phi_{t}: M \rightarrow M$ the flow along the gradient $\nabla_{g} f$. To each critical point $p \in \mathfrak{c}$, associate the stable and unstable cells

$$
S_{p}=\left\{x \in M \mid \lim _{t \rightarrow \infty} \Phi_{t}(x)=p\right\}, \quad U_{p}=\left\{x \in M \mid \lim _{t \rightarrow-\infty} \Phi_{t}(x)=p\right\} .
$$

Finally, orient $S_{p}$ and $U_{p}$ so that the orientation of $U_{p} \times S_{p}$ agrees with that of $X$ at $p$.

One formulation of Morse theory is that every cohomology class $c \in H^{*}(M ; \mathbb{R})$ can be expressed in the form

$$
c=\sum_{p \in \mathfrak{c}}\left\langle c, U_{p}\right\rangle S_{p}
$$

where $\left\langle c_{p}, U_{p}\right\rangle$ denotes the natural pairing, and we regard $S_{p}$ as a cohomology class via Poincaré duality. In other words, the stable and unstable cells form dual bases in cohomology.

To establish equation (1.3), it is useful to recast it as an equation in the cohomology of the product $X \times X$. Namely, each cohomology class $k \in H^{*}(X \times X ; \mathbb{R})$ can be regarded as an integral kernel providing an endomorphism

$$
\Phi_{k}: H^{*}(X ; \mathbb{R}) \longrightarrow H^{*}(X ; \mathbb{R}), \quad \Phi_{k}(c)=p_{2 !}\left(p_{1}^{*}(c) \cap k\right),
$$

where we use Poincaré duality to integrate. Then equation (1.3) follows from the identity of cohomology classes

$$
\Delta_{X}=\sum_{p \in \mathfrak{c}} U_{p} \times S_{p}
$$

where $\Delta_{X} \subset X \times X$ is the diagonal, and hence provides the identity endomorphism. Finally, to establish equation (1.4), one observes that the gradient flow of the Morse function

$$
f \circ p_{1}-f \circ p_{2}: X \times X \longrightarrow \mathbb{R}
$$

provides a homotopy between the diagonal $\Delta_{X}$ and the sum of external products $\sum_{p \in \mathfrak{c}} U_{p} \times S_{p}$.

All in all, a pleasant aspect of the above argument is that it makes no reference to an arbitrary cohomology class $c \in H^{*}(X ; \mathbb{R})$, but only involves highly structured cohomology classes such as the diagonal $\Delta_{X}$ and the sum of external products $\sum_{p \in \mathfrak{c}} U_{p} \times S_{p}$. Furthermore, we do not need to know a precise relation between cohomology classes $k \in H^{*}(X \times X ; \mathbb{R})$ and arbitrary endomorphisms $\Phi: H^{*}(X ; \mathbb{R}) \rightarrow H^{*}(X ; \mathbb{R})$, only that there is a linear map

$$
H^{*}(X \times X ; \mathbb{R}) \longrightarrow \text { End } H^{*}(X ; \mathbb{R}), \quad k \longmapsto \Phi_{k} .
$$

Turning from vector spaces to the setting of linear categories, one finds many examples of the above argument. Most prominently, there is Beilinson's universal resolution [9] of coherent sheaves on projective space $\mathbb{P}^{n}$ by vector bundles. It suffices once and for all to introduce the Koszul resolution of the structure sheaf $\mathcal{O}_{\Delta_{\mathbb{P}} n}$ of the diagonal $\Delta_{\mathbb{P}^{n}} \subset \mathbb{P}^{n} \times \mathbb{P}^{n}$. Then for any coherent sheaf on $\mathbb{P}^{n}$, convolution with the Koszul resolution produces the desired resolution by vector bundles. What results is a concrete description of all coherent sheaves in terms of the quiver of constituent vector bundles in the Koszul resolution.

Our sketched evidence for the recollement pattern of diagrams (1.1) and (1.2) applies the above version of Morse theory in the setting of linear categories. We consider the product Weinstein manifold $M^{\mathrm{op}} \times M$ where we write $M^{\mathrm{op}}$ to denote the opposite symplectic structure. The formalism of bimodules should allow us to view Lagrangian branes $L \subset M^{\text {op }} \times M$ as endofunctors. In particular, the diagonal brane $\Delta_{M} \subset M^{\mathrm{op}} \times M$ should represent the identity 
functor. The endofunctors of adjunctions appearing in the exact triangles of (1.2) should also be represented by natural correspondences.

A familiar but key observation is that the geometry of a neighborhood of the diagonal $\Delta_{M}$ looks like the geometry of the cotangent bundle $T^{*} M$. We highlight a useful elaboration: the geometry of natural correspondences should match the geometry of the conormal bundles to the coisotropic unstable cells.

\section{Weinstein manifolds}

Most of the material of this section is well-known and available in many beautiful sources [18, 19, 20, 75]. Unless otherwise stated, we will assume that all manifolds are real analytic, and all subsets and maps are subanalytic $[11,66]$.

\subsection{Basic notions}

Definition 2.1. An exact symplectic manifold $(M, \theta)$ is a manifold $M$ with a one-form $\theta$ such that $\omega=d \theta$ is a symplectic form. We use the term Liouville form to refer to $\theta$.

One defines the Liouville vector field $Z$ by the formula $i_{Z} \omega=\theta$. The symplectic form $\omega$ is an eigenvector for the Lie derivative $L_{Z} \omega=i_{Z} d \omega+d i_{Z} \omega=0+d \theta=\omega$. An exact symplectic manifold is equivalently a triple $(M, \omega, Z)$ consisting of a manifold $M$ with symplectic form $\omega$ and fixed vector field $Z$ such that $L_{Z} \omega=\omega$.

We will always assume that $M$ is complete with respect to $Z$ in the sense that $Z$ integrates for all time to provide an expanding action

$$
\Phi_{t}: \mathbb{R}_{+} \times M \longrightarrow M \text {. }
$$

Definition 2.2. A subset $A \subset M$ is said to be conic if it is invariant under the expanding action in the sense that $\Phi_{t}(A)=A$, for all $t \in \mathbb{R}_{+}$.

Example 2.3. Any manifold $X$ provides an exact symplectic manifold $\left(T^{*} X, \theta_{X}\right)$ given by the cotangent bundle $\pi_{X}: T^{*} X \rightarrow X$ equipped with its canonical exact structure $\theta_{X}$. The Liouville vector field $Z_{X}$ generates the standard linear scaling $\Phi_{X, t}$ along the fibers of $\pi_{X}$.

More generally, any conic open subset $A \subset T^{*} X$ in particular the complement of the zero section $T^{*} X \backslash X$, provides an exact symplectic manifold $\left(A,\left.\theta_{X}\right|_{A}\right)$.

By a Morse function $h: M \rightarrow \mathbb{R}$, we will always mean an exhausting (proper and bounded below) function whose critical points are nondegenerate and finite in number. A vector field $V$ is gradient-like with respect to $h$ if away from the critical points of $h$, we have $d h(V)>0$, and in some neighborhood of the critical points, $V$ is the gradient of $h$ with respect to some Riemannian metric.

Definition 2.4. By a Weinstein manifold, we will mean an exact symplectic manifold $(M, \theta)$ that admits a Morse function $h: M \rightarrow \mathbb{R}$ such that the Liouville vector field $Z$ is gradient-like with respect to $h$.

Example 2.5. A Weinstein cell is a Weinstein manifold $(M, \theta)$ such that $\theta$ has a single zero. It follows that any Morse function $h: M \rightarrow \mathbb{R}$ such that the Liouville vector field $Z$ is gradient-like with respect to $h$ will have a single critical point which is a minimum.

Example 2.6. Suppose $X$ is a compact manifold. Choose a generic Morse function $f_{X}: X \rightarrow \mathbb{R}$ and Riemannian metric $g$, and let $\nabla_{g} f_{X}$ denote the resulting gradient of $f_{X}$. We obtain a fiberwise linear function $F_{X}: T^{*} X \rightarrow \mathbb{R}$ by setting $F_{X}(x, \xi)=\xi\left(\left.\nabla_{g} f_{X}\right|_{x}\right)$.

Then for $\epsilon>0$ sufficiently small, the pair $\left(T^{*} X, \theta_{X}+\epsilon d F_{X}\right)$ forms a Weinstein manifold, exhibited by the Morse function $h=g+\pi_{X}^{*} f_{X}: T^{*} X \rightarrow \mathbb{R}$ given by $h(x, \xi)=|\xi|_{g}^{2}+f_{X}(x)$. 


\subsection{Cell decompositions}

For the statements of this section, in addition to the previously mentioned works, we recommend the excellent sources $[12,13]$.

Let $(M, \theta)$ be a complete exact symplectic manifold with Liouville vector field $Z$ and expanding action

$$
\Phi_{t}: \mathbb{R}_{+} \times M \longrightarrow M .
$$

Definition 2.7. The critical locus $\mathfrak{c} \subset M$ is the zero-locus of the exact structure

$$
\mathfrak{c}=\left\{x \in M|\theta|_{T_{x} M}=0\right\} .
$$

The core $K \subset M$ is the subset

$$
K=\left\{x \in M \mid \lim _{t \rightarrow \infty} \Phi_{t}(x) \in \mathfrak{c}\right\} .
$$

The ether $E \subset M$ is the complement

$$
E=M \backslash K \text {. }
$$

Remark 2.8. All three of the above subsets are evidently conic, and the expanding action on the complement $M \backslash \mathfrak{c}$, and in particular the ether $E$, is free.

The critical locus $\mathfrak{c} \subset M$ is equivalently the zero-locus of the Liouville vector field $Z$, or the fixed points of the expanding action $\Phi_{t}$.

Example 2.9. For the exact symplectic manifold $\left(T^{*} B, \theta_{B}\right)$, the core and critical locus are the zero section $\mathfrak{c}_{B}=K_{B}=B$, and the ether is its complement $E_{B}=T^{*} B \backslash B$.

More generally, for any conic open subset $A \subset T^{*} B$ and resulting exact symplectic manifold $\left(A,\left.\theta_{B}\right|_{A}\right)$, its critical locus and core are the intersection $\mathfrak{c}=K=A \cap B$, and its ether is the complement $E=A \cap\left(T^{*} B \backslash B\right)$.

Now suppose $(M, \theta)$ is a Weinstein manifold. Then the critical locus $\mathfrak{c} \subset M$ consists of the finitely many critical points of a Morse function $h: M \rightarrow \mathbb{R}$ for which $Z$ is gradient-like.

Definition 2.10. Given a Weinstein manifold $(M, \theta)$, to each critical point $p \in \mathfrak{c}$, we associate the stable and unstable manifolds

$$
c_{p}=\left\{x \in M \mid \lim _{t \rightarrow \infty} \Phi_{t}(x)=p\right\}, \quad C_{p}=\left\{x \in M \mid \lim _{t \rightarrow-\infty} \Phi_{t}(x)=p\right\},
$$

Lemma 2.11. Given a Weinstein manifold $(M, \theta)$, for each critical point $p \in \mathfrak{c}$, the stable manifold $c_{p}$ is an isotropic cell and the unstable manifold $C_{p}$ is a coisotropic cell.

The coisotropic cells provide a partition

$$
M=\coprod_{p \in \mathfrak{c}} C_{p}
$$

while the isotropic cells partition the core

$$
K=\coprod_{p \in \mathfrak{c}} c_{p} \subset M
$$

We will always perturb our Liouville form $\theta$ to be generic. Then it follows that the partition by coisotropic cells is a Whitney stratification. 
Lemma 2.12. Given a Weinstein manifold $(M, \theta)$, the core $K \subset M$ is compact and its inclusion is a homotopy-equivalence.

We will use the coisotropic cells to view the finite critical locus $\mathfrak{c} \subset M$ as a poset: we say critical points $p, q \in \mathfrak{c}$ satisfy $p \leq q$ if and only if

$$
\bar{C}_{p} \cap C_{q} \neq \varnothing .
$$

In particular, maxima $p \in \mathfrak{c}$ correspond to closed coisotropic cells $C_{p} \subset M$. Subsets $\mathfrak{s} \subset \mathfrak{c}$ such that $p \in \mathfrak{s}$ and $q \in \mathfrak{c}$, with $q \leq p$, implies $q \in \mathfrak{s}$ correspond to open unions

$$
M_{\mathfrak{s}}=\coprod_{p \in \mathfrak{s}} C_{p} \subset M
$$

\subsection{Markings}

Let us first list some useful notions about conic sets in a complete exact symplectic manifold $(M, \theta)$.

Note that the Liouville flow $\Phi_{t}: \mathbb{R}_{+} \times M \rightarrow M$ is free on the ether $E \subset M$ and so the quotient $E / \mathbb{R}_{+}$is a manifold. Furthermore, since $\theta$ vanishes on the Liouville vector field $Z$, and the Lie derivative satisfies $L_{Z} \theta=\theta$, it makes sense to ask whether $\theta$ is positive, zero or negative on a tangent vector to $E / \mathbb{R}_{+}$. It is easily checked that $\operatorname{ker}(\theta) \subset T\left(E / \mathbb{R}_{+}\right)$provides a canonical co-oriented contact structure.

Definition 2.13. Let $(M, \theta)$ be a complete exact symplectic manifold.

(1) The projectivization of $M$ is the contact manifold

$$
M^{\infty}=E / \mathbb{R}_{+}
$$

equipped with its canonical contact structure $\xi=\operatorname{ker}(\theta)$.

More generally, the projectivization of a conic subset $A \subset M$ is the subset

$$
A^{\infty}=(A \cap E) / \mathbb{R}_{+} \subset M^{\infty} .
$$

(2) The cone over a subset $A^{\infty} \subset M^{\infty}$ is the conic subset

$$
\text { с } A^{\infty}=\left\{x \in E \mid \lim _{t \rightarrow \infty} \Phi_{t}(x) \in A^{\infty}\right\} \subset M .
$$

Remark 2.14. When $(M, \theta)$ is a Weinstein manifold, its projectivization $M^{\infty}$ is compact and contactomorphic to any level set $h^{-1}(r) \subset M$ of a compatible Morse function $h: M \rightarrow \mathbb{R}$, for $r$ greater than all critical values.

Next let us list some useful notions about closed sets.

Definition 2.15. Let $(M, \theta)$ be a complete exact symplectic manifold.

(1) The compactification at infinity of $M$ is the manifold with boundary

$$
\bar{M}=((M \times(0, \infty]) \backslash(K \times\{\infty\})) / \mathbb{R}_{+} \simeq M \cup M^{\infty} .
$$

More generally, the compactification at infinity of a closed subset $A \subset M$ is the closure

$$
\bar{A} \subset \bar{M} \text {. }
$$

(2) The boundary at infinity of a closed subset $A \subset M$ is the frontier

$$
\partial^{\infty} A=\bar{A} \backslash A \subset M^{\infty}=\bar{M} \backslash M .
$$


Remark 2.16. If $A \subset M$ is closed and conic, then our notation agrees in that $A^{\infty}=\partial^{\infty} A$ as subsets of $M^{\infty}$.

Finally, we introduce the notion of a characteristic cone $\Lambda \subset M$, which could alternatively be called a "noncompact skeleton". Along with satisfying natural properties stated in the definition below, it will be a not necessarily smooth subvariety. By a subvariety $Y \subset M$, we mean nothing more than a subanalytic subset of $M$ (or if more generality is desired, a definable subset within some o-minimal context). We will only be interested in $Y$ as a topological subspace of $M$, rather than with any of its potential algebraic aspects. In particular, $Y$ admits a Whitney stratification by submanifolds of $M$, and following standard convention, we say $Y$ is isotropic if the symplectic form vanishes when restricted to each stratum.

Definition 2.17. By a marked exact symplectic manifold $(M, \theta, \Lambda)$, we will mean an exact symplectic manifold $(M, \theta)$ together with a closed conic isotropic subvariety $\Lambda \subset M$ containing the core $K \subset \Lambda$. We use the term characteristic cone to refer to $\Lambda$.

By a marked Weinstein manifold $(M, \theta, \Lambda)$, we will mean a marked exact symplectic manifold such that the underlying exact symplectic manifold $(M, \theta)$ is a Weinstein manifold.

Remark 2.18. Given a marked exact symplectic manifold $(M, \theta, \Lambda)$, we can alternatively encode the characteristic cone $\Lambda \subset M$ by taking its projectivization $\Lambda^{\infty} \subset M^{\infty}$. We recover the characteristic cone $\Lambda \subset M$ by taking the union of the core and the cone over the projectivization

$$
\Lambda=K \cup c \Lambda^{\infty} \subset M
$$

\subsection{Coisotropic cells}

Suppose $(M, \theta, \Lambda)$ is a marked Weinstein manifold. Fix a critical point $p \in \mathfrak{c}$, and consider the inclusion of the coisotropic cell $i_{p}: C_{p} \rightarrow M$.

The linear geometry of the inclusion of the coisotropic cell $i_{p}: C_{p} \rightarrow M$ provides a useful guide to keep in mind.

First, we have the normal bundle $\mathfrak{N} i_{p} \rightarrow C_{p}$ appearing in the exact sequence

$$
0 \longrightarrow T C_{p} \longrightarrow i_{p}^{*} T M \longrightarrow \mathfrak{N} i_{p} \longrightarrow 0 .
$$

Dually, we have the conormal bundle $\mathfrak{N}^{*} i_{p}=T_{C_{p}}^{*} M \rightarrow C_{p}$ appearing in the exact sequence

$$
0 \longleftarrow T^{*} C_{p} \longleftarrow i_{p}^{*} T^{*} M \longleftarrow \mathfrak{N}^{*} i_{p} \longleftarrow 0 .
$$

The symplectic form $\omega=d \theta$ provides an integrable isotropic foliation $\mathfrak{f}_{p} \subset T C_{p}$ by either taking the symplectic orthogonal $\mathfrak{f}_{p}=\left(T C_{p}\right)^{\perp}$ or equivalently, the symplectic partner $i_{\mathfrak{f}_{p}} \omega=$ $\mathfrak{N}^{*} i_{p}$. Thus the symplectic form identifies the partial flags

$$
\mathfrak{f}_{p} \smile T C_{p} \smile i_{p}^{*} T M, \quad \mathfrak{N}^{*} i_{p} \smile T_{\mathfrak{f}_{p}}^{*} M \hookrightarrow i_{p}^{*} T^{*} M,
$$

where $T_{\mathfrak{f}_{p}}^{*} M \subset i_{p}^{*} T^{*} M$ denotes the subbundle annihilating $\mathfrak{f}_{p} \subset T C_{p} \subset i_{p}^{*} T M$. Dually, it identifies the quotient sequences

$$
\mathfrak{f}_{p}^{*} T^{*} C_{p} \ll-i_{p}^{*} T^{*} M, \quad \mathfrak{N} i_{p} \ll-i_{p}^{*} T M / \mathfrak{f}_{p} \ll-i_{p}^{*} T M .
$$

Now consider the Hamiltonian reduction diagram

$$
M_{p} \stackrel{q_{p}}{\longleftarrow} C_{p} \stackrel{i_{p}}{\longrightarrow} M
$$


where $i_{p}$ is the inclusion of the coisotropic cell, and $q_{p}$ is the quotient by the integrable isotropic foliation $\mathfrak{f}_{p} \subset T C_{p}$ determined by $i_{p}$. We use the term Hamiltonian reduction diagram with the following constructions in mind.

To fix notation, suppose $\operatorname{dim} C_{p}=n+k$ where $\operatorname{dim} M=2 n$. Then we can choose $(n-k)$ local coordinates in a neighborhood of $p$ such that the intersection of $C_{p}$ with the neighborhood is their zero-locus. Using the Liouville flow $\Phi_{t}$ we can extend the local coordinates to $(n-k)$ functions in a neighborhood of $C_{p}$ such that $C_{p}$ is their zero-locus. These $(n-k)$ functions provide a moment map for an action of $\mathbb{R}^{k}$ such that $M_{p}$ is the quotient symplectic manifold. To confirm that the leaf space is indeed a manifold, and hence a symplectic manifold, it suffices to check that the foliation is fibered (or admits local slices). Observe that this is true locally in $C_{p}$, in particular in a neighborhood of $p$. Then observe that the leaves of the foliation are preserved by the Liouville flow $\Phi_{t}$, and the flow of any neighborhood of $p$ exhausts all of $C_{p}$.

With the preceding in hand, the rest of the following assertion is evident by construction.

Lemma 2.19. We have a natural marked Weinstein cell $\left(M_{p}, \theta_{p}, \Lambda_{p}\right)$ characterized by

$$
q_{p}^{*} \theta_{p}=\left.\theta\right|_{C_{p}}, \quad \Lambda_{p}=q_{p}\left(\Lambda \cap C_{p}\right) .
$$

Remark 2.20. In the case when $\Lambda^{\infty}=\varnothing$ so that $\Lambda=K$, we have $\Lambda_{p}=q_{p}\left(K \cap C_{p}\right)$. If in addition $p \in \mathfrak{c}$ is maximal, so that $C_{p} \subset M$ is closed, we have $K \cap C_{p}=\{p\}$, and hence $\Lambda_{p}=\left\{q_{p}(p)\right\}$, and so $\Lambda_{p}^{\infty}=\varnothing$.

\subsection{Recollement cone}

We continue with $(M, \theta, \Lambda)$ a marked Weinstein manifold.

Fix a critical point $p \in \mathfrak{c}$, and return to the Hamiltonian reduction diagram

$$
M_{p} \stackrel{q_{p}}{\longleftarrow} C_{p} \stackrel{i_{p}}{\longrightarrow} M
$$

where $i_{p}$ is the inclusion of the coisotropic cell, and $q_{p}$ is the quotient by the integrable isotropic foliation $\mathfrak{f}_{p} \subset T C_{p}$ determined by $i_{p}$.

Recall the natural marked Weinstein cell $\left(M_{p}, \theta_{p}, \Lambda_{p}\right)$ characterized by

$$
q_{p}^{*} \theta_{p}=\left.\theta\right|_{C_{p}}, \quad \Lambda_{p}=q_{p}\left(\Lambda \cap C_{p}\right) .
$$

Let us observe that the inverse-image

$$
\Lambda_{\tilde{p}}=q_{p}^{-1}\left(\Lambda_{p}\right) \subset C_{p}
$$

is a conic isotropic subvariety such that

$$
\Lambda \cap C_{p} \subset \Lambda_{\tilde{p}}
$$

Definition 2.21. The local recollement cone $\Lambda_{p+} \subset M$ is the conic isotropic subvariety

$$
\Lambda_{p+}=\Lambda \cup \Lambda_{\tilde{p}} \subset M .
$$

The global recollement cone $\Lambda_{+} \subset M$ is the conic isotropic subvariety

$$
\Lambda_{+}=\Lambda \cup \coprod_{p \in \mathfrak{c}} \Lambda_{p+} \subset M .
$$

Remark 2.22. In the spirit of the adjunctions to come, one could note that we can rewrite the definition of $\Lambda_{\tilde{p}} \subset C_{p}$ in the evidently equivalent forms

$$
\Lambda_{\tilde{p}}=q_{p}^{-1} q_{p}\left(\Lambda \cap C_{p}\right)=i_{p} q_{p}^{-1} q_{p} i_{p}^{-1}(\Lambda) \subset C_{p} .
$$




\subsection{Open submanifolds}

We continue with $(M, \theta, \Lambda)$ a marked Weinstein manifold.

Suppose that $p \in \mathfrak{c}$ is maximal, so that the coisotropic cell $i_{p}: C_{p} \rightarrow M$ is closed. It will be useful to introduce some further structure on its normal geometry.

Definition 2.23. Given a marked Weinstein manifold $\left(M, \theta, \Lambda^{\infty}\right)$, and a maximal critical point $p \in \mathfrak{c}$, a defining function $m_{p}: M \rightarrow[0,1]$ for the corresponding closed coisotropic cell $C_{p} \subset M$, is a subanalytic function such that:

1) $m_{p}^{-1}(0)=C_{p}$

2) $d m_{p}(Z) \leq 0$,

3) $0,1 \in[0,1]$ are the only critical values of $m_{p}$,

4) there is an open subset $U \subset M$ containing $\mathfrak{c} \backslash\{p\} \subset M$ such that $U \subset m_{p}^{-1}(1)$,

5) there is a compact subset $W \subset M$ such that $d m_{p}(Z)=0$ over the complement $M \backslash W$.

Lemma 2.24. Defining functions always exist.

Proof. This is easily obtained from the basic properties of subanalytic functions $[11,66]$.

We will say that a subset $\mathfrak{s} \subset \mathfrak{c}$ is open if $p \in \mathfrak{s}$ and $q \in \mathfrak{c}$ with $q \leq p$ implies $q \in \mathfrak{s}$. Open subsets $\mathfrak{s} \subset \mathfrak{c}$ correspond to open unions of coisotropic cells

$$
M_{\mathfrak{s}}=\coprod_{p \in \mathfrak{s}} C_{p}
$$

Lemma 2.25. For any open subset $\mathfrak{s} \subset \mathfrak{c}$, we obtain a natural marked Weinstein manifold $\left(M_{\mathfrak{s}}, \theta_{\mathfrak{s}}, \Lambda_{\mathfrak{s}}\right)$ by restriction

$$
\theta_{\mathfrak{s}}=\left.\theta\right|_{M_{\mathfrak{s}}}, \quad \Lambda_{\mathfrak{s}}=\Lambda \cap M_{\mathfrak{s}} .
$$

Proof. We must produce a Morse function $h_{\mathfrak{s}}: M_{\mathfrak{s}} \rightarrow \mathbb{R}$ such that $Z_{\mathfrak{s}}=\left.Z\right|_{M_{\mathfrak{s}}}$ is gradient-like with respect to $h_{\mathfrak{s}}$. By induction, it suffices to assume that $\mathfrak{c} \backslash \mathfrak{s}$ is a single maximal critical point $p$, so the corresponding coisotropic cell $C_{p} \subset M$ is closed.

Let $h: M \rightarrow \mathbb{R}$ be a Morse function such that $Z$ is gradient-like with respect to $h$. Choose a defining function $m_{p}: M \rightarrow[0,1]$ for the closed coisotropic cell $C_{p}=M \backslash M_{\mathfrak{s}}$. Consider the new function

$$
h_{\mathfrak{s}}=h+1 / m_{p}: M_{\mathfrak{s}} \longrightarrow \mathbb{R} .
$$

By construction, we have

$$
d h_{\mathfrak{s}}\left(Z_{\mathfrak{s}}\right)=d h(Z)-d m_{p}(Z) / m_{p}^{2} \geq 0
$$

with equality if and only if $d h=0$ hence if and only if we are at a point of $\mathfrak{s}$. Furthermore, there is a neighborhood of $\mathfrak{s}$ on which $d m_{p}=0$, and so since $Z$ is gradient-like with respect to $h$, we conclude $Z_{\mathfrak{s}}$ is gradient-like with respect to $h_{\mathfrak{s}}$.

Remark 2.26. In the case when $\Lambda^{\infty}=\varnothing$ so that $\Lambda=K$, we have $\Lambda_{\mathfrak{s}}=K \cap M_{\mathfrak{s}}$. Note that the core $K_{\mathfrak{s}} \subset M_{\mathfrak{s}}$ is contained in $K \cap M_{\mathfrak{s}}$, with equality if and only if $M_{\mathfrak{s}}=M$.

\section{$3 \quad$ Fukaya category}

In this section, we survey the construction and basic properties of the stable infinitesimal Fukaya category of a Weinstein manifold $[41,42,43,52,55]$. 


\subsection{Background structures}

Let $(M, \theta)$ be a Weinstein manifold. We briefly review the standard additional data needed to consider Lagrangian branes and the Fukaya category.

We will work with compatible almost complex structures $J \in \operatorname{End}(T M)$ that are invariant under dilations near infinity. The corresponding Riemannian metrics present $M$ near infinity as a metric cone over the projectivization $M^{\infty}$. The space of all such compatible almost complex structures is nonempty and convex.

Given an almost complex structure $J \in \operatorname{End}(T M)$, we can speak about the complex canonical line bundle $\kappa_{M}=\left(\wedge^{\operatorname{dim} M / 2} T^{\mathrm{hol}} M\right)^{-1}$. A bicanonical trivialization $\eta$ is an identification of the tensor-square $\kappa_{M}^{\otimes 2}$ with the trivial complex line bundle. The obstruction to a bicanonical trivialization is twice the first Chern class $2 c_{1}(M) \in H^{2}(M, \mathbf{Z})$, and all bicanonical trivializations form a torsor over the gauge group $\operatorname{Map}\left(M, S^{1}\right)$. Forgetting the specific almost complex structure $J \in \operatorname{End}(T M)$, we will use the term bicanonical trivialization to refer to a section of bicanonical trivializations over the space of compatible almost complex structures.

Any symplectic manifold $M$ is canonically oriented, or in other words, after choosing a Riemannian metric, the structure group of $T M$ is the special orthogonal group. A spin structure $\sigma$ is a further lift of the structure group to the spin group. The obstruction to a spin structure is the second Stiefel-Whitney class $w_{2}(M) \in H^{2}(M, \mathbf{Z} / 2 \mathbf{Z})$, and all spin structures form a torsor over the group $H^{1}(M, \mathbf{Z} / 2 \mathbf{Z})$.

Definition 3.1. A Weinstein target $(M, \theta, \eta, \sigma)$ is a Weinstein manifold $(M, \theta)$ together with a bicanonical trivialization $\eta$ and spin structure $\sigma$. We will often suppress mention of the latter structures when they are fixed throughout.

\subsection{Lagrangian branes}

By an exact Lagrangian submanifold $L \subset M$, we mean a closed but not necessarily compact submanifold of dimension $\operatorname{dim} M / 2$ such that the restriction $\left.\theta\right|_{L}$ is an exact one-form, so in particular, $\left.\omega\right|_{L}=0$ where $\omega=d \theta$.

To ensure reasonable behavior near infinity, we place two assumptions on our exact Lagrangian submanifolds $L \subset M$. First, we insist that the compactification $\bar{L} \subset \bar{M}$ is a subanalytic subset. Along with other nice properties, this implies the following two facts:

1. The boundary at infinity $\partial^{\infty} L \subset M^{\infty}$ is an isotropic subvariety.

2. For $h: M \rightarrow \mathbb{R}$ a Morse function such that $Z$ is gradient-like with respect to $h$, there is a real number $r>0$ such that the restricted function

$$
h: L \cap h^{-1}(r, \infty) \longrightarrow \mathbb{R}
$$

has no critical points.

Second, we also assume the existence of a perturbation $\psi$ that moves $L$ to a nearby exact Lagrangian submanifold that is tame (in the sense of [63]) with respect to a conic metric.

Definition 3.2. Fix a field $k$.

A brane structure on an exact Lagrangian submanifold $L \subset M$ is a three-tuple $(\mathcal{E}, \alpha, b)$ consisting of a flat finite-dimensional $k$-vector bundle $\mathcal{E} \rightarrow L$, along with a grading $\alpha$ (with respect to the given compatible class of bicanonical trivializations) and a pin structure $b$.

A Lagrangian brane in $M$ is a four-tuple $(L, \mathcal{E}, \alpha, b)$ of an exact Lagrangian submanifold $L \subset M$ equipped with a brane structure $(\mathcal{E}, \alpha, b)$. When there is no chance for confusion, we often write $L$ alone to signify the Lagrangian brane.

The objects of the Fukaya category $\mathrm{Ob} F(M)$ comprise all Lagrangian branes $L \subset M$. 
Here is a brief reminder on what a grading and pin structure entail. First, consider the bundle of Lagrangian planes $\mathcal{L}_{\mathrm{ag}_{M}} \rightarrow M$, and the squared phase map

$$
\bar{\alpha}: \mathcal{L a g}_{M} \longrightarrow \mathbf{C}^{\times}, \quad \bar{\alpha}(\mathcal{L})=\eta\left(\wedge^{\operatorname{dim} M / 2} \mathcal{L}\right)^{2} .
$$

Given a Lagrangian submanifold $L \subset M$, we obtain the restricted map

$$
\bar{\alpha}: L \longrightarrow \mathbf{C}^{\times}, \quad \bar{\alpha}(x)=\bar{\alpha}\left(T_{x} L\right) .
$$

A grading of $L$ is a lift

$$
\alpha: L \longrightarrow \mathbf{C}, \quad \bar{\alpha}=\exp \circ \alpha .
$$

The obstruction to a grading is the Maslov class $[\bar{\alpha}] \in H^{1}(L, \mathbf{Z})$, and all gradings form a torsor over the group $H^{0}(L, \mathbf{Z})$.

Second, and recall that the (positive) pin group $\operatorname{Pin}^{+}(n)$ is the double cover of the orthogonal group $O(n)$ with center $\mathbf{Z} / 2 \mathbf{Z} \times \mathbf{Z} / 2 \mathbf{Z}$. A pin structure $b$ on a Riemannian manifold $L$ of dimension $n$ is a lift of the structure group of $T L$ along the map

$$
\operatorname{Pin}^{+}(n) \longrightarrow O(n) \text {. }
$$

The obstruction to a pin structure is the second Stiefel-Whitney class $w_{2}(L) \in H^{2}(L, \mathbf{Z} / 2 \mathbf{Z})$, and all possible pin structures form a torsor over the group $H^{1}(L, \mathbf{Z} / 2 \mathbf{Z})$.

\subsection{Intersections}

Graded linear spans of intersection points provide the morphisms in the Fukaya category $F(M)$. Given a finite collection of Lagrangians branes $L_{0}, \ldots, L_{d} \in \mathrm{Ob} F(M)$, we must perturb them so that their intersections occur in some bounded domain. To organize the perturbations, we recall the inductive notion of a fringed set.

A fringed set $R_{1} \subset \mathbb{R}_{+}$is any interval of the form $(0, r)$ for some $r>0$. A fringed set $R_{d+1} \subset \mathbb{R}_{+}^{d+1}$ is a subset satisfying the following:

1. $R_{d+1}$ is open in $\mathbb{R}_{+}^{d+1}$.

2. Under the projection $\pi: \mathbb{R}^{d+1} \rightarrow \mathbb{R}^{d}$ forgetting the last coordinate, the image $\pi\left(R_{d+1}\right)$ is a fringed set.

3. If $\left(r_{1}, \ldots, r_{d}, r_{d+1}\right) \in R_{d+1}$, then $\left(r_{1}, \ldots, r_{d}, r_{d+1}^{\prime}\right) \in R_{d+1}$ for $0<r_{d+1}^{\prime}<r_{d+1}$.

A Hamiltonian function $H: M \rightarrow \mathbb{R}$ is said to be controlled if near infinity it is equal to a conical coordinate. Given a finite collection of Lagrangians branes $L_{0}, \ldots, L_{d} \in \mathrm{Ob} F(M)$, and controlled Hamiltonian functions $H_{0}, \ldots, H_{d}$, we may choose a fringed set $R \subset \mathbb{R}^{d+1}$ such that for $\left(\delta_{d}, \ldots, \delta_{0}\right) \in R$, there is a compact region $W \subset M$ such that for any $i \neq j$, we have

$$
\varphi_{H_{i}, \delta_{i}}\left(\bar{L}_{i}\right) \cap \varphi_{H_{j}, \delta_{j}}\left(\bar{L}_{j}\right) \quad \text { lies in } W .
$$

By a further compactly supported Hamiltonian perturbation, we may also arrange so that the intersections are transverse.

We consider finite collections of Lagrangian branes $L_{0}, \ldots, L_{d} \in \mathrm{Ob} F(M)$ to come equipped with such perturbation data, with the brane structures $\left(\mathcal{E}_{i}, \alpha_{i}, b_{i}\right)$ and taming perturbations $\psi_{i}$ transported via the perturbations. 
Definition 3.3. Given a finite collection of Lagrangian branes $L_{0}, \ldots, L_{d} \in \mathrm{Ob} F(M)$, for branes $L_{i}, L_{j}$ with $i<j$, the graded vector space of morphisms between them is the direct sum

$$
\operatorname{hom}_{F(M)}\left(L_{i}, L_{j}\right)=\bigoplus_{p \in \psi_{i}\left(\varphi_{H_{i}, \delta_{i}}\left(L_{i}\right)\right) \cap \psi_{j}\left(\varphi_{H_{j}, \delta_{j}}\left(L_{j}\right)\right)} \mathcal{H o m}\left(\left.\mathcal{E}_{i}\right|_{p},\left.\mathcal{E}_{j}\right|_{p}\right)[-\operatorname{deg}(p)]
$$

where the integer $\operatorname{deg}(p)$ denotes the Maslov grading at the intersection.

It is worth emphasizing that the salient aspect of the above perturbation procedure is the relative position of the perturbed branes rather than their absolute position. The following informal viewpoint can be a useful mnemonic to keep the conventions straight. In general, we always think of morphisms as "propagating forward in time". Thus to calculate the morphisms $\operatorname{hom}_{F(M)}\left(L_{0}, L_{1}\right)$, we have required that $L_{0}, L_{1}$ are perturbed near infinity so that $L_{1}$ is further in the future than $L_{0}$. But what is important is not that they are both perturbed forward in time, only that $L_{1}$ is further along the timeline than $L_{0}$. So for example, we could perturb $L_{0}, L_{1}$ near infinity in the opposite direction as long as $L_{0}$ is further in the past than $L_{1}$.

It is also worth noting the basic asymmetry of morphisms devolving from this definition. Because of the directionality of perturbations near infinity, there is no general comparison of $\operatorname{hom}_{F(M)}\left(L_{0}, L_{1}\right)$ and $\operatorname{hom}_{F(M)}\left(L_{1}, L_{0}\right)$ unlike when the branes are compact and a Calabi-Yau relation holds.

\subsection{Compositions}

Signed counts of pseudoholomorphic polygons provide the differential and higher composition maps of the $A_{\infty}$-structure of the Fukaya category $F(M)$. We use the following approach of Sikorav [63] (or equivalently, Audin-Lalonde-Polterovich [5]) to ensure that the relevant moduli spaces are compact, and hence the corresponding counts are finite.

First, a Weinstein manifold $(M, \theta)$ equipped with a compatible almost complex structure conic near infinity is tame in the sense of [63]. To see this, it is easy to derive an upper bound on its curvature and a positive lower bound on its injectivity radius.

Next, given a finite collection of branes $L_{0}, \ldots, L_{d} \in \mathrm{Ob} F(M)$, denote by $L$ the union of their perturbations $\psi_{i}\left(\varphi_{H_{i}, \delta_{i}}\left(L_{i}\right)\right)$ as described above. By construction, the intersection of $L$ with the region $M \backslash W$ is a tame submanifold (in the sense of [63]). Namely, there exists $\rho_{L}>0$ such that for every $x \in L$, the set of points $y \in L$ of distance $d(x, y) \leq \rho_{L}$ is contractible, and there exists $C_{L}$ giving a two-point distance condition $d_{L}(x, y) \leq C_{L} d(x, y)$ whenever $x, y \in L$ with $d(x, y)<\rho_{L}$.

Now, consider a fixed topological type of pseudoholomorphic map

$$
u:(D, \partial D) \longrightarrow(M, L)
$$

Assume that all $u(D)$ intersect a fixed compact region, and there is an a priori area bound Area $(u(D))<A$. Then as proven in [63], one has compactness of the moduli space of such maps $u$. In fact, one has a diameter bound (depending only on the given constants) constraining how far the image $u(D)$ can stretch from the compact set.

In the situation at hand, for a given $A_{\infty}$-structure constant, we must consider pseudoholomorphic maps $u$ from polygons with labeled boundary edges. In particular, all such maps $u$ have image intersecting the compact set given by a single intersection point. The area of the image $u(D)$ can be expressed as the contour integral

$$
\operatorname{Area}(u(D))=\int_{u(\partial D)} \theta
$$


Since each of the individual Lagrangian branes making up $L$ is exact, the contour integral only depends upon the integral of $\theta$ along minimal paths between intersection points. Thus such maps $u$ satisfy an a priori area bound. We conclude that for each $A_{\infty}$-structure constant, the moduli space defining the structure constant is compact, and its points are represented by maps $u$ with image bounded by a fixed distance from any of the intersection points.

Definition 3.4. Given a finite collection of Lagrangian branes $L_{0}, \ldots, L_{d} \in \mathrm{Ob} F(M)$, the composition map

$$
m^{d}: \operatorname{hom}_{F(M)}\left(L_{0}, L_{1}\right) \otimes \cdots \otimes \operatorname{hom}_{F(M)}\left(L_{d-1}, L_{d}\right) \rightarrow \operatorname{hom}_{F(M)}\left(L_{0}, L_{d}\right)[2-d]
$$

is defined as follows. Consider elements $p_{i} \in \operatorname{hom}\left(L_{i}, L_{i+1}\right)$, for $i=0, \ldots, d-1$, and $p_{d} \in$ $\operatorname{hom}\left(L_{0}, L_{d}\right)$. Then the coefficient of $p_{d}$ in $m^{d}\left(p_{0}, \ldots, p_{d-1}\right)$ is defined to be the signed sum over pseudoholomorphic maps from a disk with $d+1$ counterclockwise cyclically ordered marked points mapping to the $p_{i}$ and corresponding boundary arcs mapping to the perturbations of $L_{i+1}$. Each map contributes according to the holonomy of its boundary, where adjacent perturbed components $L_{i}$ and $L_{i+1}$ are glued with $p_{i}$.

\subsection{Coherence}

In the preceding sections, we have described the objects, morphisms, and compositions of the Fukaya category $F(M)$. As explained in the fundamental sources $[22,55]$, there are a large number of details to organize to be sure to obtain an honest $A_{\infty}$-category. In particular, calculations require branes be in general position, and hence must be invariant under suitable perturbations. In the setting of noncompact branes, we have additional perturbations near infinity to keep track of. In particular, at first pass, the constructions given only provide what might be called a compatible collection of directed $A_{n}$-categories, for all $n$. Here $A_{n}$ denotes Stasheff's operad of partial associative operations [64, 65], and we use the term directed as in [55]. The former arises since we only define finitely many composition coefficients at one time, and the latter since our perturbations near infinity are directed "forward in time". To confirm the coherence of the definitions, one can appeal to a refined version of the well-known invariance of Floer calculations under Hamiltonian isotopies. We include a brief discussion here (largely borrowed from [42]) to explain the key ideas behind this approach.

Let $h: M \rightarrow \mathbb{R}$ be a Morse function compatible with the Weinstein structure, in particular, providing a conical coordinate near infinity.

Definition 3.5. By a one-parameter family of closed (but not necessarily compact) submanifolds (without boundary) of $M$, we mean a closed submanifold

$$
\mathfrak{L} \hookrightarrow \mathbb{R} \times M
$$

satisfying the following:

1. The restriction of the projection $p_{\mathbb{R}}: \mathbb{R} \times M \rightarrow \mathbb{R}$ to the submanifold $\mathfrak{L}$ is nonsingular.

2. There is a real number $r>0$, such that the restriction of the product $p_{\mathbb{R}} \times h: \mathbb{R} \times M \rightarrow \mathbb{R} \times \mathbb{R}$ to the subset $\{h>r\} \cap \mathfrak{L}$ is proper and nonsingular.

3. There is a compact interval $[a, b] \hookrightarrow \mathbb{R}$ such that the restriction of the projection $p_{M}$ : $\mathbb{R} \times M \rightarrow M$ to the submanifold $p_{\mathbb{R}}^{-1}([\mathbb{R} \backslash[a, b]) \cap \mathfrak{L}$ is locally constant.

Remark 3.6. Conditions (1) and (2) will be satisfied if the restriction of the projection $\bar{p}_{\mathbb{R}}$ : $\mathbb{R} \times \bar{M} \rightarrow \mathbb{R}$ to the closure $\overline{\mathfrak{L}} \hookrightarrow \bar{M}$ is nonsingular as a stratified map, but the weaker condition stated is a useful generalization. It implies in particular that the fibers $\mathfrak{L}_{s}=p_{\mathbb{R}}^{-1}(s) \cap \mathfrak{L} \hookrightarrow M$ are all diffeomorphic, but imposes no requirement that their boundaries at infinity should all be homeomorphic as well. 
Definition 3.7. By a one-parameter family of tame Lagrangian branes in $M$, we mean a oneparameter family of closed submanifolds $\mathfrak{L} \hookrightarrow \mathbb{R} \times M$ such that the fibers $\mathfrak{L}_{s}=p_{\mathbb{R}}^{-1}(s) \cap \mathfrak{L} \hookrightarrow M$ also satisfy:

1. The fibers $\mathfrak{L}_{s}$ are exact tame Lagrangians with respect to the symplectic structure and any almost complex structure conical near infinity.

2. The fibers $\mathfrak{L}_{s}$ are equipped with a locally constant brane structure $\left(\mathcal{E}_{s}, \alpha_{s}, b_{s}\right)$ with respect to the given background classes.

Remark 3.8. Note that if we assume that $\mathfrak{L}_{0}$ is an exact Lagrangian, then $\mathfrak{L}_{s}$ being an exact Lagrangian is equivalent to the family $\mathfrak{L}$ being given by the flow $\varphi_{H_{s}}$ of the vector field of a time-dependent Hamiltonian $H_{s}: M \rightarrow \mathbb{R}$. Note as well that a brane structure consists of topological data, so can be transported unambiguously along the fibers of such a family.

Remark 3.9. It is important for various applications of the theory of this section that we consider one-parameter families of tame Lagrangian branes $\mathfrak{L} \hookrightarrow \mathbb{R} \times M$ such that the fiber branes $\mathfrak{L}_{s} \subset M$ are not constant near infinity. Thus in general the fiber branes will not be isomorphic objects of $F(M)$, though we will see that their Floer-pairing with respect to certain test branes will be invariant.

The rest of this section will be devoted to the following statement of Floer invariance. It is the basic instance (going beyond the foundational results of $[22,55]$ ) of the general pattern that confirms $F(M)$ is a well-defined $A_{\infty}$-category.

Proposition 3.10. Suppose $\mathfrak{L}_{s}$ is a one-parameter family of tame Lagrangian branes in $M$. Suppose $L^{\prime}$ is a fixed test brane which is disjoint from $\mathfrak{L}_{s}$ near infinity for all s. Suppose $\mathfrak{L}_{s}$ is transverse to $L^{\prime}$ except for finitely many points.

Then for any $a, b$ with $\mathfrak{L}_{a}$ and $\mathfrak{L}_{b}$ transverse to $L^{\prime}$, the Floer chain complexes $\operatorname{CF}\left(\mathfrak{L}_{a}, L^{\prime}\right)$ and $\mathrm{CF}\left(\mathfrak{L}_{b}, L^{\prime}\right)$ are quasi-isomorphic.

Before proving the proposition in general, it is convenient to first prove the following special case.

Lemma 3.11. Suppose $\mathfrak{L}_{s}$ is one-parameter family of tame Lagrangian branes in $M$. Suppose $L^{\prime}$ is a fixed test object which is disjoint from $\mathfrak{L}_{s}$ near inf inity for all $s$.

Fix $s_{0}$ and assume $\mathfrak{L}_{s_{0}}$ is transverse to $L^{\prime}$. Then there is an $\epsilon>0$ so that for all $s_{1} \epsilon$ $\left(s_{0}-\epsilon, s_{0}+\epsilon\right)$, the Floer chain complexes $\mathrm{CF}\left(\mathfrak{L}_{s_{0}}, L^{\prime}\right)$ and $\mathrm{CF}\left(\mathfrak{L}_{s_{1}}, L^{\prime}\right)$ are quasi-isomorphic.

Proof. By our assumptions on the tame behavior (in the sense of [63]) of $\mathfrak{L}_{s_{0}}$ and $L^{\prime}$ near infinity, the moduli spaces giving the differential of $\mathrm{CF}\left(\mathfrak{L}_{s_{0}}, L^{\prime}\right)$ are compact. This follows from the a priori $C^{0}$-bound: there is some $r_{0} \gg 0$, such that no disk in the moduli space leaves the region $h<r_{0}$, where $h: M \rightarrow \mathbb{R}$ is a compatible Morse function.

Choose some $r_{1}>r_{0}$. Then for very small $\epsilon>0$ and any $s_{1} \in\left(s_{0}-\epsilon, s_{0}+\epsilon\right)$, we may decompose the motion $\mathfrak{L}_{s_{0}} \rightsquigarrow \mathfrak{L}_{s_{1}}$ into two parts: first, a motion $\mathfrak{L}_{s_{0}} \rightsquigarrow L$ supported in the region $h>r_{1}$; and then second, a compactly supported motion $L \rightsquigarrow \mathfrak{L}_{s_{1}}$. We must show that each of the above two motions leads to a quasi-isomorphism.

First, for the motion $\mathfrak{L}_{s_{0}} \rightsquigarrow L$, since we have not changed $\mathfrak{L}_{s_{0}}$ or $L^{\prime}$ in the region $h<r_{0}$, the same a priori $C^{0}$-bounds of [63] hold (they only depend on the Lagrangians in the region $\left.h<r_{0}\right)$, and the pseudoholomorphic strips for the pair $\left(\mathfrak{L}_{s_{0}}, L^{\prime}\right)$ and for the pair $\left(L, L^{\prime}\right)$ are in fact exactly the same (we could perversely attach "wild" non-intersecting ends to either and it would not make a difference.) Thus we can take the continuation map to be the identity.

Second, the motion $L \rightsquigarrow \mathfrak{L}_{s_{1}}$ is compactly supported, so standard PDE techniques provide a continuation map. 
Remark 3.12. In the proof of the lemma, one should probably not use the term "continuation map" for the quasi-isomorphism associated to the first motion $\mathfrak{L}_{s_{0}} \rightsquigarrow L$. Rather, it is an example of the more general setup of parameterized moduli spaces. In the above setting, one can obtain a uniform $C^{0}$-bound over the family, so the parameterized moduli space is compact, and hence one can apply standard cobordism arguments to prove the matrix coefficients at the initial and final time are the same.

Proof of Proposition 3.10. By the previous lemma, it suffices to show that for any $s_{0}$ with $\mathfrak{L}_{s_{0}}$ not (necessarily) transverse to $L^{\prime}$, there is a small $\epsilon>0$ such that the Floer chain complexes $\mathrm{CF}\left(\mathfrak{L}_{s_{0}-\epsilon}, L^{\prime}\right)$ and $\mathrm{CF}\left(\mathfrak{L}_{s_{0}+\epsilon}, L^{\prime}\right)$ are quasi-isomorphic.

To see this, fix a compatible Morse function $h: M \rightarrow \mathbb{R}$, and a bump function $b: \mathbb{R} \rightarrow \mathbb{R}$ such that the composition $b \circ h: M \rightarrow \mathbb{R}$ is identically 0 near infinity and 1 on a compact set containing all of the critical points of $h$ and (possibly non-transverse) intersection points $\mathfrak{L}_{s_{0}} \cap L^{\prime}$

Let $H_{s}: M \rightarrow \mathbb{R}$ be a (time-dependent) Hamiltonian giving the motion $\mathfrak{L}_{s}$. The product Hamiltonian $\tilde{H}_{s}=(b \circ h) \cdot H_{s}: M \rightarrow \mathbb{R}$ gives a family $\tilde{\mathfrak{L}}_{s}$ through the base object $\mathfrak{L}_{s_{0}}$ satisfying: (1) $\tilde{\mathfrak{L}}_{s}$ is transverse to $L^{\prime}$ whenever $\left|s-s_{0}\right|$ is small and nonzero, and (2) $\tilde{\mathfrak{L}}_{s}$ is equal to $\mathfrak{L}_{s_{0}}$ near infinity. Therefore since the motion of $\tilde{\mathfrak{L}}_{s}$ is compactly supported, standard PDE techniques provide a continuation map giving a quasi-isomorphism between $\mathrm{CF}\left(\tilde{\mathfrak{L}}_{s_{0}-\epsilon}, L^{\prime}\right)$ and $\mathrm{CF}\left(\tilde{\mathfrak{L}}_{s_{0}+\epsilon}, L^{\prime}\right)$, for small enough $\epsilon>0$.

Finally, returning to the bump function $b$, one can construct motions $\mathfrak{L}_{s_{0}-\epsilon} \rightsquigarrow \tilde{\mathfrak{L}}_{s_{0}-\epsilon}$ and $\tilde{\mathfrak{L}}_{s_{0}+\epsilon} \rightsquigarrow \mathfrak{L}_{s_{0}+\epsilon}$ which are supported near infinity and thus in particular always transverse to $L^{\prime}$. Thus we may apply the previous lemma to obtain quasi-isomorphisms between $\operatorname{CF}\left(\mathfrak{L}_{s_{0}-\epsilon}, L^{\prime}\right)$ and $\operatorname{CF}\left(\tilde{\mathfrak{L}}_{s_{0}-\epsilon}, L^{\prime}\right)$, and similarly, between $\operatorname{CF}\left(\tilde{\mathfrak{L}}_{s_{0}+\epsilon}, L^{\prime}\right)$ and $\operatorname{CF}\left(\mathfrak{L}_{s_{0}+\epsilon}, L^{\prime}\right)$. Putting together the above, we obtain a quasi-isomorphism between $\operatorname{CF}\left(\mathfrak{L}_{s_{0}-\epsilon}, L^{\prime}\right)$ and $\operatorname{CF}\left(\mathfrak{L}_{s_{0}+\epsilon}, L^{\prime}\right)$.

Remark 3.13. The above proposition (which is a condensed form of arguments of [41, 43] and appears explicitly in [42] for cotangent bundles) is closely related to Question 1.3 of Oh's paper [44] which asks whether a homology-level continuation map constructed by a careful limiting argument with PDE techniques is induced by a chain-level morphism. While we have not investigated this, it is not hard to believe that the quasi-isomorphism of the above proposition provides the desired lift.

\subsection{Stabilization}

It is convenient to work interchangeably with small idempotent-complete pre-triangulated $k$ linear $A_{\infty}$-categories $[22,55]$ when thinking about Fukaya categories and small stable idempotent-complete $k$-linear $\infty$-categories $[34,35]$ when thinking about abstract constructions. They have equivalent homotopy theories, and we lose nothing by going back and forth. In what follows, all of the specific assertions we will use can be found in [10].

Definition 3.14. Let $\mathcal{C}, \mathcal{C}^{\prime}$ be stable $\infty$-categories. A functor $F: \mathcal{C} \rightarrow \mathcal{C}^{\prime}$ is said to be

(1) continuous if it preserves coproducts,

(2) proper if it preserves compact objects,

(3) exact if it preserves zero objects and exact triangles (equivalently, finite colimits).

It is convenient to work alternatively within two related $k$-linear contexts.

Definition 3.15. We denote by $\mathrm{St}_{k}$ the symmetric monoidal $\infty$-category of stable presentable $k$-linear $\infty$-categories with morphisms continuous functors. The monoidal unit is the $\infty$-category Mod $k$ of $k$-chain complexes. 
We denote by st $_{k}$ the symmetric monoidal $\infty$-category of small stable idempotent-complete $k$-linear $\infty$-categories with morphisms exact functors. The monoidal unit is the $\infty$-category Perf $k$ of perfect $k$-chain complexes.

Definition 3.16. The big stabilization $\operatorname{Mod} \mathcal{C} \in \mathrm{St}_{k}$ of a small $k$-linear $\infty$-category $\mathcal{C}$ is the stable presentable $k$-linear differential graded category of $A_{\infty}$-right modules

$\mathcal{C}^{\mathrm{op}} \longrightarrow \operatorname{Mod} k$.

The small stabilization $\operatorname{Perf} \mathcal{C} \in \mathrm{st}_{k}$ is the small $k$-linear full $\infty$-subcategory of Mod $\mathcal{C}$ consisting of compact objects (summands of finite colimits of representable objects).

Lemma 3.17. For a small $k$-linear $\infty$-category $\mathcal{C}$, the Yoneda embedding is fully faithful

$$
\mathcal{Y}: \mathcal{C} \longrightarrow \operatorname{Perf} \mathcal{C}, \quad \mathcal{Y}_{L}(P)=\operatorname{hom}_{\mathcal{C}}(P, L) .
$$

If $\mathcal{C} \in \mathrm{st}_{k}$, then the Yoneda embedding is an equivalence.

Corollary 3.18. For a small $k$-linear $\infty$-category $\mathcal{C}$, forming its stabilization canonically commutes with forming its opposite category

$$
\operatorname{Perf}\left(\mathcal{C}^{\text {op }}\right) \simeq \operatorname{Perf}(\mathcal{C})^{\text {op }}
$$

Lemma 3.19. Forming big stabilizations is a faithful symmetric monoidal functor

$$
\operatorname{Mod}: \mathrm{st}_{k} \longrightarrow \mathrm{St}_{k} \text {. }
$$

The monoidal dual of $\mathcal{C} \in \mathrm{st}_{k}$ is the opposite category $\mathcal{C}^{\mathrm{op}}$.

The monoidal dual of $\operatorname{Mod} \mathcal{C} \in \mathrm{St}_{k}$ is the restricted opposite category $\operatorname{Mod}\left(\mathcal{C}^{\mathrm{op}}\right)$.

Remark 3.20. We can recover $\mathcal{C} \in \mathrm{st}_{k}$ from $\operatorname{Mod} \mathcal{C} \in \mathrm{St}_{k}$ by passing to compact objects $\mathcal{C}=(\operatorname{Mod} \mathcal{C})^{c}$. The image of the morphism $\operatorname{Mod}: \operatorname{hom}_{\mathrm{st}_{k}}\left(\mathcal{C}, \mathcal{C}^{\prime}\right) \rightarrow \operatorname{hom}_{\mathrm{St}_{k}}\left(\operatorname{Mod} \mathcal{C}, \operatorname{Mod} \mathcal{C}^{\prime}\right)$ comprises proper functors.

Corollary 3.21. For $\mathcal{C}, \mathcal{C}^{\prime} \in \mathrm{st}_{k}$, there are canonical equivalences

$$
\begin{aligned}
& \mathcal{C}^{\mathrm{op}} \otimes \mathcal{C}^{\prime} \stackrel{\sim}{\longrightarrow} \operatorname{hom}_{\mathrm{st}_{k}}\left(\mathcal{C}, \mathcal{C}^{\prime}\right) \\
& \operatorname{Mod}\left(\mathcal{C}^{\mathrm{op}} \otimes \mathcal{C}^{\prime}\right) \simeq \operatorname{Mod}\left(\mathcal{C}^{\mathrm{op}}\right) \otimes \operatorname{Mod}\left(\mathcal{C}^{\prime}\right) \stackrel{\sim}{\longrightarrow} \operatorname{hom}_{\mathrm{St}_{k}}\left(\operatorname{Mod} \mathcal{C}, \operatorname{Mod} \mathcal{C}^{\prime}\right) .
\end{aligned}
$$

Definition 3.22. The perfect Fukaya category $\operatorname{Perf} F(M)$ is the small stabilization of $F(M)$.

The stable Fukaya category $\operatorname{Mod} F(M)$ is the big stabilization of $F(M)$.

\subsection{Singular support}

While calculations among Lagrangian branes reflect quantum topology, we nevertheless have access to their underlying Lagrangian submanifolds. We take advantage of this in the following definition.

Definition 3.23. Fix an object $L \in \operatorname{Perf} F(M)$.

(1) The null locus $n(L) \subset M$ is the conic open subset of points $x \in M$ for which there exists a conic open set $U \subset M$ containing $x$ such that we have the vanishing

$$
\operatorname{hom}_{F(M)}(L, P) \simeq 0, \quad \text { for any } \quad P \in F(M) \text { with } \quad P \subset U, \quad \partial^{\infty} P \subset U^{\infty} .
$$


(2) The singular support $\operatorname{ss}(L) \subset M$ is the conic closed complement

$$
\operatorname{ss}(L)=M \backslash n(L) .
$$

Remark 3.24. For cotangent bundles, under the equivalence of branes and constructible sheaves recalled in the next section, the above notion of singular support for branes coincides with the traditional notion of singular support of sheaves.

Definition 3.25. Let $(M, \theta, \Lambda)$ be a marked Weinstein manifold.

We define the full subcategory $\operatorname{Perf}_{\Lambda} F(M) \subset \operatorname{Perf} F(M)$ to comprise objects $L \in \operatorname{Perf} F(M)$ with singular support satisfying $\operatorname{ss}(L) \subset \Lambda$.

Lemma 3.26. $\operatorname{Perf}_{\Lambda} F(M)$ is a small stable idempotent-complete k-linear $\infty$-category. If $\Lambda_{1} \subset \Lambda_{2}$, then $\operatorname{Perf}_{\Lambda_{1}} F(M) \subset \operatorname{Perf}_{\Lambda_{2}} F(M)$, and $\operatorname{Perf} F(M)=\cup_{\Lambda} \operatorname{Perf}_{\Lambda} F(M)$.

Proof. The singular support condition is clearly preserved by extensions and summands.

In the remainder of this section, we explain (without proof) how to calculate the projectivization of the singular support. By induction using recollement, this provides a complete picture of the singular support. We will not need this material for any further developments, but include it to help orient the reader.

Fix a finite collection of Lagrangian branes $L_{i} \in F(M)$, for $i \in I$, and let $V=\cup_{i \in I} L_{i} \subset M$ denote the Lagrangian subvariety given by their union.

Lemma 3.27. The boundary at infinity $\partial^{\infty} V \subset M^{\infty}$ is a closed Legendrian subvariety.

Proof. By dilation, we can contract $V \subset M$ to a conical Lagrangian subvariety $V^{c} \subset M$. Then we need only observe that $\partial^{\infty} V=\partial^{\infty} V^{c}$.

Let $\partial_{s m}^{\infty} V \subset \partial^{\infty} V$ denote the smooth locus. Given a point $x \in \partial_{s m}^{\infty} V$, we can find a small Legendrian sphere $S(x) \subset M^{\infty}$ centered at $x$, and simply linked around $\partial_{s m}^{\infty} V$. Then we can find a Lagrangian brane $B(x) \subset M$ diffeomorphic to a ball, and with boundary at infinity $\partial^{\infty} B(x)=S(x)$. The particular grading on $B(x)$ will play no role.

Proposition 3.28. For an object $L \in \operatorname{Perf} F(M)$ in the perfect envelope of the finite collection $L_{i} \in F(M)$, for $i \in I$, the projectivization of its singular support $\operatorname{ss}(L)^{\infty} \subset M^{\infty}$ is the closure of the subset

$$
\left\{x \in \partial_{s m}^{\infty} V \mid \operatorname{hom}_{F(M)}(L, B(x)) \not 0\right\} .
$$

Corollary 3.29. The projectivization of the singular support $\operatorname{ss}(L)^{\infty} \subset M^{\infty}$ is a Legendrian subvariety.

\subsection{Cotangent bundles}

We briefly remind the reader of the equivalence between branes in a cotangent bundle and constructible sheaves on the base manifold.

Let $X$ be a compact manifold with cotangent bundle $\pi: T^{*} X \rightarrow X$ and projectivization $\pi^{\infty}: T^{\infty} X \rightarrow X$. For simplicity, let us assume that $X$ is equipped with an orientation and spin structure. Then as explained for instance in Example 2.6, we can view $T^{*} X$ as a Weinstein manifold with a canonically trivial canonical bundle and canonical spin structure.

Definition 3.30. Let $\operatorname{Sh}(X)$ denote the differential graded category of complexes of sheaves on $X$ with constructible cohomology. 
Definition 3.31. Given an object $\mathcal{F} \in \operatorname{Sh}(X)$, we write ss $\mathcal{F} \subset T^{*} X$ for its singular support, and $\mathrm{ss}^{\infty} \mathcal{F} \subset T^{\infty} X$ for the projectivization of its singular support.

Given a Whitney stratification $\mathcal{S}=\left\{X_{\alpha}\right\}_{\alpha \in A}$, we define its conormal bundle and projectivized conormal bundle to be the unions

$$
T_{\mathcal{S}}^{*} X=\coprod_{\alpha \in A} T_{X_{\alpha}}^{*} X \subset T^{*} X, \quad T_{\mathcal{S}}^{\infty} X=\coprod_{\alpha \in A} T_{X_{\alpha}}^{\infty} X \subset T^{\infty} X .
$$

Definition 3.32. Suppose $\Lambda \subset T^{*} X$ is a conical Lagrangian subvariety. Let $\operatorname{Sh}_{\Lambda}(X) \subset \operatorname{Sh}(X)$ denote the full subcategory of complexes of sheaves with ss $\mathcal{F} \subset \Lambda$.

Suppose $\mathcal{S}=\left\{X_{\alpha}\right\}_{\alpha \in A}$ is a Whitney stratification of $X$. Let $\operatorname{Sh}_{\mathcal{S}}(X) \subset \operatorname{Sh}(X)$ denote the full subcategory of complexes of sheaves with $\mathcal{S}$-constructible cohomology.

Lemma 3.33. For a Whitney stratification $\mathcal{S}$, we have $\operatorname{Sh}_{\mathcal{S}}(X)=\operatorname{Sh}_{T_{\mathcal{S}}^{*} X}(X)$.

Now let $i: Y \rightarrow X$ be a locally closed submanifold with frontier $\partial Y=\bar{Y} \backslash Y$. On the one hand, we have the standard and costandard extensions $i_{*} k_{Y}, i_{!} k_{Y} \in \operatorname{Sh}(X)$.

On the other hand, we have corresponding Lagrangian branes constructed as follows. Choose a non-negative function $m: X \rightarrow \mathbb{R}_{\geq 0}$ with zero-set precisely $\partial Y \subset X$.

Definition 3.34. We define the standard and costandard Lagrangians $L_{Y *}, L_{Y !} \in F\left(T^{*} X\right)$ to be the fiberwise translations

$$
L_{Y *}=\Gamma_{d \log m}+T_{Y}^{*} X, \quad L_{Y !}=-\Gamma_{d \log m}+T_{Y}^{*} X
$$

equipped with the orientation bundle or $Y$, and canonical gradings and spin structures.

We have the following from $[41,42,43]$.

Theorem 3.35. There is a canonical equivalence

$$
\mu_{X}: \operatorname{Sh}(X) \stackrel{\sim}{\longrightarrow} \operatorname{Perf} F\left(T^{*} X\right)
$$

such that $\mu_{X}\left(i_{*} k_{Y}\right) \simeq L_{Y *}, \mu_{X}\left(i_{!} k_{Y}\right) \simeq L_{Y !}$.

Furthermore, we have $\mathrm{ss}^{\infty} \mathcal{F}=\mathrm{ss}^{\infty} \mu_{X}(\mathcal{F})$, and hence for a conical Lagrangian subvariety $\Lambda \subset T^{*} X$ containing the zero section, $\mu_{X}$ restricts to an equivalencez

$$
\mu_{X}: \operatorname{Sh}_{\Lambda}(X) \stackrel{\sim}{\longrightarrow} \operatorname{Perf}_{\Lambda} F\left(T^{*} X\right) .
$$

There are various extensions of the above result to noncompact manifolds $X$, but we will only call upon the following expected generalization in support of the anticipated recollement pattern sketched in the next section.

Suppose $X$ is a manifold whose noncompactness is concentrated at single conical end. In other words, we have a manifold $Y$ with boundary $\partial Y$ such that $\bar{Y}=Y \amalg \partial Y$ is compact, and an identification

$$
X \simeq \bar{Y} \coprod_{\partial Y}(\partial Y \times[0, \infty))
$$

We can equivalently assume $X$ is equipped with a Morse function $f_{X}: X \rightarrow \mathbb{R}$ and Riemannian metric $g$ such that the flow of the gradient $\nabla_{g} f_{X}$ exhibits the conical end as a metric product.

Consider the fiberwise linear function $F_{X}: T^{*} X \rightarrow \mathbb{R}$ defined by $F_{X}(x, \xi)=\xi\left(\left.\nabla_{g} f_{X}\right|_{x}\right)$, and the Morse function $h=g+\pi_{X}^{*} f_{X}: T^{*} X \rightarrow \mathbb{R}$ defined by $h(x, \xi)=|\xi|_{g}^{2}+f_{X}(x)$. For $\epsilon>0$ sufficiently small, the pair $\left(T^{*} X, \theta_{X}+\epsilon d F_{X}\right)$ should form a Weinstein manifold exhibited by the Morse function $h$. 
Now suppose in addition that $\mathcal{S}=\left\{X_{\alpha}\right\}_{\alpha \in A}$ is a Whitney stratification with strata $X_{\alpha} \subset X$ that are conical near the end. In other words, we assume that the strata $X_{\alpha}$ are transverse to $\partial Y$ inside of $X$, and the above identification restricts to an identification

$$
X_{\alpha}=\left(X_{\alpha} \cap \bar{Y}\right) \coprod_{\left(X_{\alpha} \cap \partial Y\right)}\left(\left(X_{\alpha} \cap \partial Y\right) \times[0, \infty)\right) .
$$

We can equivalently assume the strata are invariant under the flow of the gradient of our Morse function along the conical end.

Now let $i: Y \rightarrow X$ be a union of strata. On the one hand, we have the standard and costandard extensions $i_{*} k_{Y}, i_{!} k_{Y} \in \operatorname{Sh}_{\mathcal{S}}(X)$.

On the other hand, we have the corresponding standard and costandard branes $L_{Y *}, L_{Y !} \in$ $F\left(T^{*} X\right)$ constructed with a non-negative function $m: X \rightarrow \mathbb{R}_{\geq 0}$ invariant under the flow of the gradient of our Morse function along the conical end.

With only slight modifications, the arguments used to establish Theorem 3.35 should provide the following proposition. To realize it, one could embed $X$ as an open submanifold of a compact manifold $Z$ and interpret all of the calculations to take place there. In particular, one could extend all sheaves by zero off of $X$ and work with the corresponding branes in $T^{*} Z$ as prescribed by Theorem 3.35. Thanks to our perturbation framework and diameter bounds on disks, the relevant geometry should be equivalent to that within $T^{*} X$.

Now we expect there is a canonical functor

$$
\mu_{X}: \operatorname{Sh}_{\mathcal{S}}(X) \longrightarrow \operatorname{Perf} F\left(T^{*} X\right)
$$

such that $\mu_{X}\left(i_{*} k_{Y}\right) \simeq L_{Y *}, \mu_{X}\left(i_{!} k_{Y}\right) \simeq L_{Y !}$

Remark 3.36. We will return to the above in the special situation when $X$ itself is a Weinstein manifold, with $f_{X}$ a Morse function compatible with its Liouville vector field, and $\mathcal{S}$ the stratification by coisotropic cells.

\section{Adjunctions for branes}

Due to the technical demands of the material, this section will be more informal than the others. We outline the expected recollement pattern of diagrams (1.1) and (1.2), only sketching the constructions and results that we anticipate should hold.

\subsection{Bimodules via correspondences}

Definition 4.1. Let $(M, \theta)$ be an exact symplectic manifold. The opposite exact symplectic manifold $\left(M^{\mathrm{op}},-\theta\right)$ is the same underlying manifold $M$ equipped with the negative Liouville form, and hence negative symplectic form $-\omega=-d \theta$.

When $(M, \theta)$ is equipped with background structures, we transport them by the identity to obtain background structures on $\left(M^{\mathrm{op}},-\theta\right)$. In particular, given a compatible almost complex structure $J \in \operatorname{End}(T M)$, we take $-J \in \operatorname{End}\left(T M^{\mathrm{op}}\right)$. Thus we have an identity of bicanonical line bundles $\kappa_{M}$ op $\simeq \kappa_{M}^{-1}$ and so a bicanonical trivialization for $(M, \theta)$ induces one for $\left(M^{\text {op }},-\theta\right)$.

Lemma 4.2. If $(M, \theta)$ is a Weinstein manifold, its opposite $\left(M^{\mathrm{op}},-\theta\right)$ is a Weinstein manifold with the same Liouville vector field.

Proof. If $\theta=i_{Z} \omega$, then $-\theta=i_{Z}(-\omega)$. 
Proposition 4.3. There is a canonical identification

$$
F(M) \stackrel{\sim}{\longrightarrow} F\left(M^{\mathrm{op}}\right)^{\mathrm{op}}
$$

given on Lagrangian branes by the duality

$$
L=(L, \mathcal{E}, \alpha, b) \longmapsto L^{\prime}=\left(L, \mathcal{E}^{\vee},-\alpha, b\right) .
$$

Proof. Our perturbation framework is compatible with the assertion.

Definition 4.4. Suppose $M, N$ are Weinstein targets.

A Lagrangian correspondence is an object $\mathrm{K} \in F\left(M^{\mathrm{op}} \times N\right)$. The dual correspondence $\mathrm{K}^{\prime} \in$ $F\left(N^{\mathrm{op}} \times M\right)$ is the matched object under the equivalence $F\left(M^{\mathrm{op}} \times N\right) \simeq F\left(N^{\mathrm{op}} \times M\right)^{\mathrm{op}}$.

Suppose $M, N$ are Weinstein targets, and $\mathrm{K} \in F\left(M^{\mathrm{op}} \times N\right)$ a Lagrangian correspondence. Let $L \in F(M), P \in F(N)$ be test branes.

One should be able to obtain a functor

$$
f_{\mathrm{K}}: F(M) \longrightarrow \operatorname{Mod} F(N)
$$

or equivalently a morphism

$$
f_{\mathrm{K}} \in \operatorname{hom}_{\mathrm{St}_{k}}(\operatorname{Mod} F(M), \operatorname{Mod} F(N)) \simeq \operatorname{Mod}\left(F\left(M^{\mathrm{op}}\right) \otimes F(N)\right)
$$

given by the functorial construction

$$
f_{\mathrm{K}}(L)(P)=\operatorname{hom}_{F\left(M^{\mathrm{op}} \times N\right)}\left(L^{\prime} \times P, \mathrm{~K}\right) .
$$

There are (at least) two approaches one might take to confirm that the above definition is sensible. The two approaches we will mention here should lead to homotopically equivalent theories.

On the one hand, one could adopt the geometric formalism of Lagrangian correspondences as developed by Wehrheim and Woodward [67, 68, 69, 70, 71, 72] and Ma'u [39] and count pseudoholomorphic quilts to provide the structure constants of an $A_{\infty}$-functor.

On the other hand, one should be able to adopt the algebraic formalism of bimodules. To implement this, unwinding the Yoneda embedding, one needs to know that the product on branes induces a bilinear functor

$$
F\left(M^{\mathrm{op}}\right) \times F(N) \longrightarrow F\left(M^{\mathrm{op}} \times N\right) .
$$

With this in hand, one could then define the module $f_{\mathrm{K}}(L)$ as the composition

$$
f_{\mathrm{K}}(L): F(N) \longrightarrow \operatorname{Mod} k, \quad f_{\mathrm{K}}(L)(P)=\operatorname{hom}_{F\left(M^{\mathrm{op}} \times N\right)}\left(L^{\prime} \times P, \mathrm{~K}\right) .
$$

This should have the simultaneous functoriality in $P$ to make $f_{K}(L)$ a module, and the functoriality in $L$ to make $f_{K}$ a functor. Note that establishing the existence of the product functor is much less than proving that the induced linear functor

$$
F\left(M^{\mathrm{op}}\right) \otimes F(N) \longrightarrow F\left(M^{\mathrm{op}} \times N\right)
$$

is an equivalence. Coupled with Corollary 3.21 in mind, this stronger assertion would show all functors are given by kernels, but this is not necessary for the aims outlined above. As far as we know, the existence of the product functor is not in the literature, though related homotopical algebra is available [31, 38, 49, 50] and some readers may prefer this point of view to the intricacies of Lagrangian correspondences and pseudoholomorphic quilts.

With the appropriate brane structure, the diagonal $\Delta_{M} \subset M^{\text {op }} \times M$ Lagrangian correspondence should give an endofunctor $f_{\Delta_{M}}$ of $F(M)$ canonically equivalent to the identity $\operatorname{id}_{F(M)}$. A similar assertion in parallel settings can be found in many places including $[67,68,69,70,71,72]$. 
Remark 4.5. The above integral transform formalism is tuned to constructing right adjoints. There is an alternative dual formalism suited to constructing left adjoints.

Given a Lagrangian correspondence $\mathrm{K} \in F\left(M^{\mathrm{op}} \times N\right)$, and test branes $L \in F(M), P \in F(N)$, one should be able also to obtain a functor

$$
g_{\mathrm{K}}: F(M)^{\mathrm{op}} \longrightarrow \operatorname{Mod}\left(F(N)^{\mathrm{op}}\right)
$$

given by the functorial construction

$$
g_{\mathrm{K}}(L)(P)=\operatorname{hom}_{F\left(M^{\mathrm{op}} \times N\right)}\left(\mathrm{K}, L^{\prime} \times P\right) .
$$

Note this should be contravariant in the Lagrangian correspondence K.

If the above produces a proper functor, then in turn it can be interpreted as a functor $g_{\mathrm{K}}: \operatorname{Perf} F(M) \rightarrow \operatorname{Perf} F(N)$.

With the appropriate brane structure, the diagonal $\Delta_{M} \subset M^{\text {op }} \times M$ Lagrangian correspondence should give an endofunctor $g_{\Delta_{M}}$ of $F(M)$ canonically equivalent to the identity $\operatorname{id}_{F(M)}$. (One should not expect the same brane structure on $\Delta_{M}$ to result in both functors $f_{\Delta_{M}}$ and $g_{\Delta_{M}}$ being the identity.)

\subsection{Closed cell correspondences}

Fix a maximal critical point $p \in \mathfrak{c}$, so that the corresponding coisotropic cell is closed, and consider the Hamiltonian reduction diagram

$$
M_{p} \stackrel{q_{p}}{\longleftarrow} C_{p} \stackrel{i_{p}}{\longrightarrow} M,
$$

where $i_{p}$ is the inclusion of the coisotropic cell, and $q_{p}$ is the quotient by the integrable isotropic foliation determined by $i_{p}$.

Define the closed cell correspondence

$$
\mathrm{C}_{p} \in F\left(M_{p}^{\mathrm{op}} \times M\right)
$$

to be the Lagrangian submanifold $C_{p} \subset M_{p}^{\text {op }} \times M$ equipped with a brane structure. Since we are only sketching constructions, we will not specify the details of particular brane structures throughout what follows.

We will sketch that the correspondence construction

$$
f_{\mathrm{C}_{p}}: \operatorname{Mod} F\left(M_{p}\right) \longrightarrow \operatorname{Mod} F(M)
$$

in fact should restrict to a fully faithful representable functor

$$
\mathfrak{i}: F\left(M_{p}\right) \longrightarrow F(M)
$$

Observe that the geometric composition of a Lagrangian brane $L \subset M_{p}$ with the correspondence $C_{p}$ is nothing more than the pullback $i_{p}^{-1} L \subset M$. The object $\mathfrak{i}(L)$ representing $f_{\mathrm{C}_{p}}(L)$ should be the pullback $i_{p}^{-1} L \subset M$ with an induced brane structure. This is a geometric assertion whose proof should be similar to the statement that the diagonal brane gives the identity correspondence.

To see why the functor should be fully faithful, consider the fiber $F_{p}=i_{p}^{-1}(p) \subset C_{p}$ and its cotangent bundle $T^{*} F_{p}$. We can find a small open neighborhood $\mathcal{N}\left(C_{p}\right) \subset M$ of the coisotropic cell $C_{p} \subset M$ and a symplectic identification $\mathcal{N}\left(C_{p}\right) \simeq M_{p} \times U_{p}$, where the factor $U_{p} \subset T^{*} F_{p}$ is 
a small open neighborhood of the zero section. Now observe that with this setup, the functor $\mathfrak{i}$ should be given by taking the product with the zero section

$$
F\left(M_{p}\right) \longrightarrow F\left(M_{p} \times U_{p}\right), \quad L \longmapsto L \times F_{p}
$$

Following our perturbation framework, near infinity we perturb such products by a product of a perturbation of $L$ and a perturbation of $F_{p}$. For the latter, the perturbation will be to the graph of the differential of a function on $F_{p}$ which is linear at infinity. In particular, we can take the function on $F_{p}$ to have a single critical point.

Now it should suffice to show that all disks involved in the calculation of morphisms between such products lie in $\mathcal{N}\left(C_{p}\right)$. Following for example [41, 43], this can be accomplished as follows. First, by contracting the first factor with the Liouville vector field of $M_{p}$, we can ensure arbitrarily small energy bounds on the disks. Then we can invoke [63] so that the energy bounds provide sufficient diameter bounds.

Remark 4.6. For a fixed marking $\Lambda_{p} \subset M_{p}$, consider the induced marking

$$
\Lambda_{p+}=K \cup q_{p}^{-1}\left(\Lambda_{p}\right) \subset M
$$

The functor $\mathfrak{i}$ should restrict to a functor

$$
\mathfrak{i}: F_{\Lambda_{p}}\left(M_{p}\right) \longrightarrow F_{\Lambda_{p+}}(M)
$$

Remark 4.7. One can alternatively apply the above discussion within the dual formalism of integral transforms suited to constructing left adjoints. As above, with the appropriate brane structure, the closed cell correspondence $\mathrm{C}_{p} \in F\left(M_{p}^{\mathrm{op}} \times M\right)$ should give a fully faithful functor $g_{\mathrm{C}_{p}}$ that is also equivalent to $i$. (One should not expect the same brane structure on $\mathrm{C}_{p}$ to lead to both functors $f_{\mathrm{C}_{p}}$ and $g_{\mathrm{C}_{p}}$ being the functor $\mathfrak{i}$.)

Next, there should exist a right adjoint

$$
\mathfrak{i}^{!}: \operatorname{Mod} F(M) \longrightarrow \operatorname{Mod} F\left(M_{p}\right)
$$

given by the correspondence construction $f_{\mathrm{C}_{p}^{\prime}}$ with an appropriate brane structure on $\mathrm{C}_{p}^{\prime}$. Once one knows that $\mathfrak{i}$ is given by the dual correspondence construction $g_{\mathrm{C}_{p}}$, it should be a formal consequence that its right adjoint is given by $f_{\mathrm{C}_{p}^{\prime}}$. Following patterns for constructible sheaves, we expect that $\mathfrak{i}^{!}$should in fact be proper.

Remark 4.8. One can alternatively apply the above discussion within the dual formalism of integral transforms suited to constructing left adjoints.

First, one can regard the functor $\mathfrak{i}$ as a proper continuous functor

$$
\operatorname{Mod}\left(F\left(M_{p}\right)^{\mathrm{op}}\right) \longrightarrow \operatorname{Mod}\left(F(M)^{\mathrm{op}}\right) .
$$

Then there should exist an additional adjoint $\mathfrak{i}^{*}$ given by the correspondence construction $g_{\mathrm{C}_{p}^{\prime}}$ with an appropriate brane structure on $\mathrm{C}_{p}^{\prime}$. Once one knows that $\mathfrak{i}$ is given by the correspondence construction $f_{\mathrm{C}_{p}}$, it should be a formal consequence that the additional adjoint is given by $g_{\mathrm{C}_{p}^{\prime}}$.

Moreover, following patterns for constructible sheaves, we expect that $\mathfrak{i}^{*}$ should in fact be proper. Thus in turn it could be regarded as a functor Perf $F(M) \rightarrow \operatorname{Perf} F\left(M_{p}\right)$, which should provide a left adjoint to the original functor $i$. 


\subsection{Open complements}

We continue with a maximal critical point $p \in \mathfrak{c}$, so that the corresponding coisotropic cell $C_{p} \subset M$ is closed. Now let us consider the open subset $\mathfrak{s}=\mathfrak{c} \backslash\{p\}$, and the corresponding open union of coisotropic cells

$$
M_{\mathfrak{s}}=\coprod_{q \in \mathfrak{s}} C_{q}=M \backslash C_{p}
$$

Before proceeding further, it will be convenient to pin down the choice of a defining function $m_{p}: M \rightarrow[0,1]$ for the coisotropic cell $C_{p} \subset M$ more specifically. All of what follows can be achieved by applications of the Lagrangian and coisotropic neighborhood theorems $[25,73,74]$ and the fact that $C_{p} \subset M$ is a minimal unstable cell.

First, let us return to constructions seen in the preceding section. Consider the fiber $F_{p}=$ $i_{p}^{-1}(p) \subset C_{p}$ and its cotangent bundle $T^{*} F_{p}$. We can find a small open neighborhood $\mathcal{N}\left(C_{p}\right) \subset M$ and a symplectic identification $\mathcal{N}\left(C_{p}\right) \simeq M_{p} \times U_{p}$, where the factor $U_{p} \subset T^{*} F_{p}$ is a small open neighborhood of the zero section. Refining the cell $M_{p}$ of the first factor, we can choose a Lagrangian $L_{p} \subset M_{p}$ and identifications $L_{p} \simeq \mathbb{R}^{k}$ and $T^{*} L_{p} \simeq M_{p}$, where $k=\operatorname{dim} M_{p} / 2$. Refining the normal second factor $U_{p}$, we can choose a fiber $N_{p} \subset U_{p}$ of the projection $T^{*} F_{p} \rightarrow$ $F_{p}$ and identifications $N_{p} \simeq \mathbb{R}^{n}$ and $U_{p} \simeq T^{*} N_{p}$, where $n=\operatorname{dim} M-\operatorname{dim} C_{p}$. Thus altogether, we have identifications $\mathcal{N}\left(C_{p}\right) \simeq T^{*}\left(L_{p} \times N_{p}\right) \simeq T^{*}\left(\mathbb{R}^{k} \times \mathbb{R}^{n}\right)$. Finally, we can choose the defining function $m_{p}: M \rightarrow[0,1]$ so that outside of the open neighborhood $\mathcal{N}\left(C_{p}\right) \subset M$, it is identically one, and near to $C_{p} \subset \mathcal{N}\left(C_{p}\right)$, it is simply the sum of the squares of the coordinates of the normal factor $N_{p} \simeq \mathbb{R}^{n}$.

Consider the Hamiltonian function $\log m_{p}: M_{\mathfrak{s}} \rightarrow \mathbb{R}$, and the symplectomorphisms

$$
\Xi_{\mathfrak{s} !}: M_{\mathfrak{s}} \longrightarrow M_{\mathfrak{s}}, \quad \Xi_{\mathfrak{s} *}: M_{\mathfrak{s}} \longrightarrow M_{\mathfrak{s}}
$$

resulting from the Hamiltonian flow of $\log m_{p}$ for negative unit time and unit time respectively. To ensure good behavior near infinity, it is technically useful to introduce a sufficiently small $\eta>0$ and the Hamiltonian function $\log \left(m_{p}-\eta\right): M_{\mathfrak{s},>\eta} \rightarrow \mathbb{R}$ on the domain $M_{\mathfrak{s},>\eta}=$ $\left\{x \in M_{\mathfrak{s}} \mid m_{p}(x)>\eta\right\}$, and the symplectomorphisms

$$
\Xi_{\mathfrak{s}, \eta !}: M_{\mathfrak{s},>\eta} \longrightarrow M_{\mathfrak{s},>\eta}, \quad \Xi_{\mathfrak{s}, \eta *}: M_{\mathfrak{s},>\eta} \longrightarrow M_{\mathfrak{s},>\eta}
$$

resulting from the Hamiltonian flow of $\log \left(m_{p}-\eta\right)$ for negative unit time and unit time respectively. By subanalytic theory, any subanalytic subset $Y \subset \bar{M}$ must be transverse to the closed ball $\overline{M_{\mathfrak{s}, \eta}} \subset \bar{M}$, given by the closure of the open ball $M_{\mathfrak{s}, \eta}=\left\{x \in M_{\mathfrak{s}} \mid m_{p}(x)=\eta\right\}$, for sufficiently small $\eta>0$.

Now for fixed $\dagger=$ ! or $*$, we expect that given a brane $L \in F\left(M_{\mathfrak{s}}\right)$, for all sufficiently small $\eta>0$, the pushforwards

$$
\Xi_{\mathfrak{s}, \eta \dagger}\left(L \cap M_{\mathfrak{s}, \eta}\right)
$$

are well-defined objects of $F(M)$ and all mutually diffeomorphic.

They also should all be diffeomorphic to the limit pushforward

$$
\Xi_{\mathfrak{s}, 0^{+\dagger}}(L)=\lim _{\eta \rightarrow 0^{+}} \Xi_{\mathfrak{s}, \eta \dagger}\left(L \cap M_{\mathfrak{s}, \eta}\right)
$$

which also should be a well-defined object of $F(M)$.

We expect a proof to proceed along the following lines. By construction, $\Xi_{\mathfrak{s}, \eta \dagger}\left(L \cap M_{\mathfrak{s}, \eta}\right)$ is diffeomorphic to $L$ and coincides with it on a common subset that is a deformation retract of 
each. Thus to see $\Xi_{\mathfrak{s}, \eta \dagger}\left(L \cap M_{\mathfrak{s}, \eta}\right)$ is a well-defined brane, we should check that it is closed in $M$. Suppose not: then there would be a sequence of points along the boundary at infinity of $L$ at which $d m_{p}$ is a negative scale of the contact form $\alpha$ for the boundary of $M_{\mathfrak{s}}$. By the curve selection lemma, there should be a curve $\gamma$ of such points, and hence $0>d m_{p}\left(\gamma^{\prime}\right)=c \alpha\left(\gamma^{\prime}\right)$ for some negative function $c$. But this would contradict the fact that the boundary at infinity of $L$ must be isotropic.

Now we expect that for $\dagger=!, *$, there is a fully faithful functor

$$
F\left(M_{\mathfrak{s}}\right) \longrightarrow F(M)
$$

with continuous extension

$$
\mathfrak{j}_{\dagger}: \operatorname{Mod} F\left(M_{\mathfrak{s}}\right) \longrightarrow \operatorname{Mod} F(M)
$$

given on Lagrangian branes $L \in F\left(M_{\mathfrak{s}}\right)$ by the limit

$$
\mathfrak{j}_{\dagger} L=\Xi_{\mathfrak{s}, 0^{+\dagger}}(L) .
$$

The existence of the functor $\mathfrak{j}_{\dagger}$ and the fact that it is fully faithful should all follow from some simple observations. Consider a finite collection of branes in $M_{\mathfrak{s}}$ and finite collection of $A_{\infty}$-compositions. Observe that the construction $\Xi_{\mathfrak{s}, 0^{+} \dagger}$ is compatible with our perturbation framework: near infinity, perturbing forward in time is compatible with applying $\Xi_{\mathfrak{s}, 0^{+} *}$, and backward in time with $\Xi_{\mathfrak{s}, 0^{+} !}$. Thus we can compare calculations before and after applying $\Xi_{\mathfrak{s}, 0^{+} \dagger}$ by collectively using the appropriate direction of perturbation. Next, observe that the relevant intersections and disks in all calculations are constrained to a compact subset $K \subset M_{\mathfrak{s}}$ thanks to diameter bounds depending only on a smaller compact set. Thus changes to the target and branes outside of $K \subset M_{\mathfrak{s}}$ are immaterial. In particular, since the construction $\Xi_{\mathfrak{s}, 0^{+} \dagger}$ takes place outside of $M_{\mathfrak{s}, \eta}$, for sufficiently small $\eta$, it has no effect on any calculations. Thus the extension of branes given by $\Xi_{\mathfrak{s}, 0^{+} \dagger}$ should be a fully faithful embedding.

Remark 4.9. Recall the induced markings

$$
\Lambda_{\mathfrak{s}}=\Lambda \cap M_{\mathfrak{s}} \subset M_{\mathfrak{s}}, \quad \Lambda_{p}=q_{p}\left(\Lambda \cap C_{p}\right) \subset M_{p}, \quad \Lambda_{p+}=\Lambda \cup q_{p}^{-1}\left(\Lambda_{p}\right) \subset M .
$$

The functor $\mathfrak{j}_{\dagger}$ should restrict to a functor

$$
\mathfrak{j}_{\dagger}: F_{\Lambda_{\mathfrak{s}}}\left(M_{\mathfrak{s}}\right) \longrightarrow F_{\Lambda_{p+}}(M)
$$

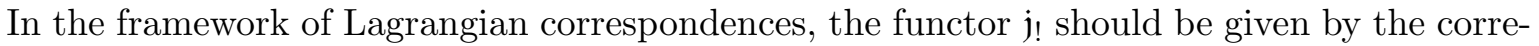
spondence construction $g_{\Gamma_{\mathfrak{s} !}}$ where we write $\Gamma_{\mathfrak{s} !} \subset M_{\mathfrak{s}}^{\mathrm{op}} \times M$ for the graph of $\Xi_{\mathfrak{s} !}$ equipped with an appropriate brane structure. This is a geometric assertion whose proof should be similar to the statement that the diagonal brane gives the identity correspondence.

Next, $\mathfrak{j}$ ! should admit a right adjoint

$$
\mathfrak{j}^{!}: \operatorname{Mod} F(M) \longrightarrow \operatorname{Mod} F\left(M_{\mathfrak{s}}\right)
$$

given by the correspondence constructions $f_{\Gamma_{\mathfrak{s} !}^{\prime}}$ with an appropriate brane structure on $\Gamma_{\mathfrak{s} !}^{\prime}$. Once one knows that $j_{\text {! }}$ is given by the dual correspondence construction $g_{\Gamma_{5 !}}$, it should be a formal consequence that its right adjoint is given by $f_{\Gamma_{s !}^{\prime}}$. Following patterns for constructible sheaves, we expect that $j^{!}$should in fact be proper. 
Remark 4.10. One can alternatively apply the above discussion within the dual formalism of integral transforms suited to constructing left adjoints.

First, the functor $\mathfrak{j}_{*}$ should be given by the correspondence construction $f_{\Gamma_{\mathfrak{s} *}}$ where we write $\Gamma_{\mathfrak{s} *} \subset M_{\mathfrak{s}}^{\text {op }} \times M$ for the graph of $\Xi_{\mathfrak{s} *}$ equipped with an appropriate brane structure.

Then there should exist an additional adjoint $j^{*}$ given by the correspondence construction $g_{\Gamma_{\mathfrak{s}}^{\prime}}$ with an appropriate brane structure on $\Gamma_{\mathfrak{s} *}^{\prime}$. Moreover, following patterns for constructible sheaves, we expect that $j^{*}$ should in fact be proper. Thus in turn it could be regarded as a functor Perf $F\left(M_{\mathfrak{s}}\right) \rightarrow \operatorname{Perf} F(M)$ which should provide a left adjoint to the original functor $\mathfrak{j}_{*}$.

\subsection{Simple identities}

We mention here some simple relations that should hold between the functors introduced in the previous sections. There should be vanishing

$$
\mathfrak{j} ! \mathfrak{i} \simeq 0: F\left(M_{p}\right) \longrightarrow F\left(M_{\mathfrak{s}}\right), \quad \mathfrak{i}^{\prime} \mathfrak{j}_{*} \simeq 0: F\left(M_{\mathfrak{s}}\right) \longrightarrow F\left(M_{p}\right)
$$

since thanks to our perturbation framework, given test branes, each of the above compositions should result in a configuration with non-intersecting branes.

Remark 4.11. Within the dual formalism of integral transforms suited to constructing left adjoints, one should similarly have identities

$$
\mathfrak{j}^{*} \mathfrak{i} \simeq 0, \quad \mathfrak{i}^{*} \mathbf{j} ! \simeq 0
$$

\subsection{Geometry of diagonal}

We continue with the previous setup: a maximal critical point $p \in \mathfrak{c}$ corresponding to a closed coisotropic cell $C_{p} \subset M$, and open subset $\mathfrak{s}=\mathfrak{c} \backslash\{p\}$ corresponding to the open complement

$$
M_{\mathfrak{s}}=\coprod_{q \in \mathfrak{s}} C_{q}=M \backslash C_{p}
$$

Let us sketch the existence of an open neighborhood $\mathcal{N}\left(\Delta_{M} \cup\left(C_{p} \times_{M_{p}} C_{p}\right)\right) \subset M^{\text {op }} \times M$, an open neighborhood $\mathcal{N}\left(T_{M}^{*} M \cup T_{C_{p}}^{*} M\right) \subset T^{*} M$, and a symplectomorphism

$$
\psi: \mathcal{N}\left(\Delta_{M} \cup\left(C_{p} \times_{M_{p}} C_{p}\right)\right) \stackrel{\sim}{\longrightarrow} \mathcal{N}\left(T_{M}^{*} M \cup T_{C_{p}}^{*} M\right),
$$

such that we have identifications

$$
\begin{aligned}
& \psi\left(\Delta_{M}\right)=T_{M}^{*} M, \quad \psi\left(C_{p} \times_{M_{p}} C_{p}\right)=T_{C_{p}}^{*} M, \\
& \psi\left(\Gamma_{\mathfrak{s} !}\right)=-\Gamma_{d \log m_{p}}, \quad \psi\left(\Gamma_{\mathfrak{s} *}\right)=\Gamma_{d \log m_{p}} .
\end{aligned}
$$

For concreteness, let us return to the identifications introduced earier. Namely, we have an open neighborhood $\mathcal{N}\left(C_{p}\right) \subset M$, and identifications

$$
\mathcal{N}\left(C_{p}\right) \simeq M_{p} \times U_{p} \simeq T^{*}\left(L_{p} \times N_{p}\right) \simeq T^{*}\left(\mathbb{R}^{k} \times \mathbb{R}^{n}\right),
$$

where $L_{p} \subset M_{p}, N_{p} \subset U_{p}$ are Lagrangian cells. Under these identifications, the coisotropic cell $C_{p}$ goes over to the coisotropic cell $T^{*} \mathbb{R}^{k} \times\left. T_{\{0\}}^{*} \mathbb{R}^{n} \simeq T^{*}\left(\mathbb{R}^{k} \times \mathbb{R}^{n}\right)\right|_{\mathbb{R}^{k} \times\{0\}}$. Furthermore, the support of $d \log m_{p}$ is contained within $\mathcal{N}\left(C_{p}\right)$. 
Let us simplify the notation and write $V=\mathbb{R}^{k} \times \mathbb{R}^{n}$, and $W=\mathbb{R}^{k} \times\{0\}$. Let us further introduce notation for elements $x, y \in V, \xi, \eta \in V^{*}$, and consider the symplectomorphism

$$
\begin{aligned}
& \left(V \times V^{*}\right)^{\mathrm{op}} \times\left(V \times V^{*}\right) \simeq\left(T^{*} V\right)^{\mathrm{op}} \times T^{*} V \stackrel{\sim}{\longrightarrow} T^{*}\left(T^{*} V\right) \simeq\left(V \times V^{*}\right) \times\left(V^{*} \times V\right), \\
& (x, \xi, y, \eta) \longmapsto \frac{1}{\sqrt{2}}(x+y, \xi+\eta, \eta-\xi, x-y) .
\end{aligned}
$$

It takes the coisotropic cell $\left(\left.T^{*} V\right|_{W}\right) \times_{T^{*} W}\left(\left.T^{*} V\right|_{W}\right)$ to the coisotropic cell $T_{\left.T^{*} V\right|_{W}}^{*}\left(T^{*} V\right)$.

Now working first near $C_{p} \times{ }_{M_{p}} C_{p} \subset M^{\mathrm{op}} \times M$, we can use the above constructions to define $\psi$ satisfying the requirements. To extend it to a neighborhood around the rest of $\Delta_{M}$, we can use the Lagrangian neighborhood theorem [73, 74].

Next define the closed cell projector

$$
\Pi_{p} \in F\left(M^{\mathrm{op}} \times M\right)
$$

to be the Lagrangian submanifold $C_{p} \times_{M_{p}} C_{p} \subset M^{\mathrm{op}} \times M$ equipped with an appropriate brane structure.

For $\dagger=!, *$, define the open complement projector

$$
\Pi_{\mathfrak{s} \dagger} \in F\left(M^{\text {op }} \times M\right)
$$

to be the Lagrangian graph $\Gamma_{\Xi \dagger} \subset M^{\mathrm{op}} \times M$ equipped with an appropriate brane structure.

Let us next sketch how inside of $F\left(M^{\mathrm{op}} \times M\right)$, we should have exact triangles of branes

$$
\Pi_{p} \longrightarrow \Delta_{M} \longrightarrow \Pi_{\mathfrak{s} *} \stackrel{[1]}{\longrightarrow}, \quad \Pi_{\mathfrak{s} !} \longrightarrow \Delta_{M} \longrightarrow \Pi_{p} \stackrel{[1]}{\longrightarrow}
$$

with the appropriate brane structures.

On the one hand, given any open neighborhood $\mathcal{N}\left(\Delta_{M}\right) \subset M^{\mathrm{op}} \times M$, and any finite number of $A_{\infty}$-compositions among the branes in the assertion of the theorem, we can arrange so that all relevant geometry lies in $\mathcal{N}\left(\Delta_{M}\right)$.

On the other hand, using the microlocalization functor

$$
\mu_{M}: \operatorname{Sh}(M) \longrightarrow F\left(T^{*} M\right)
$$

we can transport the standard exact triangles

$$
i_{i} i^{\prime} \mathcal{D}_{M} \longrightarrow \mathcal{D}_{M} \longrightarrow j_{*} j^{*} \mathcal{D}_{M} \stackrel{[1]}{\longrightarrow}, \quad j_{!} j^{!} k_{M} \longrightarrow k_{M} \longrightarrow i_{*} i^{*} k_{M} \stackrel{[1]}{\longrightarrow},
$$

where $k_{M}$ denotes the constant sheaf and $\mathcal{D}_{M}$ the Verdier dualizing sheaf, to exact triangles of branes

$$
\Pi_{p} \longrightarrow \Delta_{M} \longrightarrow \Pi_{\mathfrak{s *}} \stackrel{[1]}{\longrightarrow}, \quad \Pi_{\mathfrak{s} !} \longrightarrow \Delta_{M} \longrightarrow \Pi_{p} \stackrel{[1]}{\longrightarrow}
$$

with the appropriate brane structures.

Furthermore, we can arrange so that all relevant geometry lies in any neighborhood $\mathcal{N}\left(\Delta_{M} \cup\right.$ $\left.\left(C_{p} \times_{M_{p}} C_{p}\right)\right) \subset M^{\mathrm{op}} \times M$. Thus choosing a neighborhood and symplectomorphism as sketched above, we obtain a matching of all calculations.

It follows that any object $L \in \operatorname{Mod} F(M)$ should fit into an exact triangle

$$
\mathfrak{i i} ! L \longrightarrow L \longrightarrow \mathfrak{j}_{*} \mathfrak{j}^{*} L \stackrel{[1]}{\longrightarrow}
$$


To see this, apply the integral transform formalism to the first exact triangle of branes above to obtain an exact triangle

$$
f_{\Pi_{p}}(L) \longrightarrow L \longrightarrow f_{\Pi_{\mathfrak{s *}}}(L) \stackrel{[1]}{\longrightarrow}
$$

Then it remains to establish equivalences

$$
\begin{aligned}
& f_{\Pi_{p}} \simeq \mathfrak{i} \circ \mathfrak{i}^{!} \in \operatorname{Fun}(\operatorname{Mod} F(M), \operatorname{Mod} F(M)), \\
& f_{\Pi_{\mathfrak{s} *}} \simeq \mathfrak{j}_{*} \circ \mathfrak{j}^{!} \in \operatorname{Fun}(\operatorname{Mod} F(M), \operatorname{Mod} F(M)) .
\end{aligned}
$$

These should admit direct verification or alternatively as a non-compact variation on the theory of Lagrangian correspondences of Wehrheim and Woodward [67, 68, 69, 70, 71, 72].

Remark 4.12. The other expected triangle should result from taking the second triangle of branes above

$$
\Pi_{\mathfrak{s} !} \longrightarrow \Delta_{M} \longrightarrow \Pi_{p} \stackrel{[1]}{\longrightarrow}
$$

and applying the dual formalism of integral transforms suited to constructing left adjoints. We expect all functors should be proper, and so for any object $L \in \operatorname{Mod}\left(F(M)^{\mathrm{op}}\right)$, what should result is an exact triangle

$$
\mathfrak{j}_{!} \mathfrak{j}^{!} L \longrightarrow L \longrightarrow \mathfrak{i i}^{*} L \stackrel{[1]}{\longrightarrow}
$$

of objects of $F(M)$.

\subsection{A further identity}

We also expect to have an equivalence $\mathfrak{j}^{!} \simeq \mathfrak{j}^{*}$. To see this, we seek a natural equivalence

$$
\operatorname{hom}_{\operatorname{Perf} F\left(M_{\mathfrak{s}}\right)}\left(\mathfrak{j}^{!} L, P\right) \simeq \operatorname{hom}_{F(M)}\left(L, \mathfrak{j}_{*} P\right) \text {. }
$$

Since both sides vanish for objects of the form $L=\mathfrak{i} L^{\prime}$, it suffices to assume that $L=\mathfrak{j}$ ! $P^{\prime}$, and to establish a natural equivalence

$$
\operatorname{hom}_{F\left(M_{\mathfrak{s}}\right)}\left(P^{\prime}, P\right) \simeq \operatorname{hom}_{F(M)}\left(\mathfrak{j}_{!} P^{\prime}, \mathfrak{j}_{*} P\right) .
$$

This should follow from the compatibility of the perturbation framework with the construction of the functors.

\section{Localization}

We return now to a more traditional presentation. The constructions of this section will not depend on the recollement pattern of diagrams (1.1) and (1.2) of the introduction as sketched in the preceding section. But verifying their good properties in Section 5.4 will.

We continue to suppose throughout this section that $(M, \theta, \eta, \sigma)$ is a fixed Weinstein target, so a Weinstein manifold $(M, \theta)$ with a bicanonical trivialization $\eta$ and spin structure $\sigma$. We will also suppose as well that each of its Weinstein cells $\left(M_{p}, \theta_{p}\right)$ is equipped with a bicanonical trivialization $\eta_{p}$ and the (necessarily) trivial spin structure $\sigma_{p}$. 


\subsection{Preliminaries}

Definition 5.1. The conic topology of $M$ is the category $M_{\text {con }}$ with objects conic open subanalytic subsets of $M$ and morphisms inclusions.

Recall that st $_{k}$ denotes the $\infty$-category of small stable idempotent-complete $k$-linear $\infty$ categories.

Definition 5.2. (1) $\mathrm{A} \mathrm{st}_{k}$-valued presheaf on the conic topology of $M$ is an $\infty$-functor

$$
\mathcal{F}^{\text {pre }}: M_{\text {con }}^{\text {op }} \longrightarrow \text { st }_{k} \text {. }
$$

(2) $\mathrm{A} \mathrm{st}_{k}$-valued sheaf on the conic topology of $M$ is a continuous st $_{k}$-valued presheaf.

Definition 5.3. The sheafification $\mathcal{F}=\left(\mathcal{F}^{\text {pre }}\right)^{+}$of a presheaf $\mathcal{F}^{\text {pre }}$ is a sheaf equipped with a universal presheaf morphism

$$
\mathcal{F}^{\text {pre }} \longrightarrow \mathcal{F} \text {. }
$$

In our arguments, we will only need the following elementary functoriality. Throughout what follows, $\mathcal{F}$ always denotes a st $_{k}$-valued presheaf or sheaf on the conic topology of $M$.

Definition 5.4. Let $j: U \rightarrow M$ be the inclusion of a conic open set.

The restriction $j^{*} \mathcal{F}=\left.\mathcal{F}\right|_{U}$ is obtained by pullback along the induced functor $j: U_{\text {con }} \rightarrow M_{\text {con }}$. In other words, it assigns $j^{*} \mathcal{F}(V)=\mathcal{F}(V)$ to any conic open set $V \subset U$.

Definition 5.5. Let $\pi: M \rightarrow N$ be a conic map.

The pushforward $\pi_{*} \mathcal{F}$ is obtained by pullback along the induced functor $\pi^{-1}: N_{\text {con }} \rightarrow M_{\text {con }}$. In other words, it assigns $\pi_{*} \mathcal{F}(U)=\mathcal{F}\left(\pi^{-1}(U)\right)$ to any conic open set $U \subset N$.

Remark 5.6. Restriction evidently commutes with sheafification, while pushforward does not in general.

Remark 5.7. Note that the global sections of a presheaf or sheaf are simply its pushforward to a point.

Definition 5.8. The support of $\mathcal{F}$ is the smallest conic closed set $S \subset M$ such that $\left.\mathcal{F}\right|_{M \backslash S} \simeq 0$.

Remark 5.9. To see that the support is well-defined, note that if $S_{1}, S_{2} \subset M$ are conic closed sets such that $\left.\left.\mathcal{F}\right|_{M \backslash S_{1}} \simeq \mathcal{F}\right|_{M \backslash S_{2}} \simeq 0$, then the sheaf property implies $\left.\mathcal{F}\right|_{M \backslash\left(S_{1} \cap S_{2}\right)} \simeq 0$.

\subsection{Construction of sheaf}

Definition 5.10. Let $U \subset M$ be a conic open subset.

We define the full subcategory of $U$-null branes $\operatorname{Null}(M, U) \subset \operatorname{Perf} F(M)$ to comprise objects $L \in \operatorname{Perf} F(M)$ with singular support satisfying

$$
\operatorname{ss}(L) \cap U=\varnothing,
$$

or equivalently, null locus satisfying

$$
U \subset n(L) .
$$

Remark 5.11. As a special case of the above definition, if we start with a characteristic cone $\Lambda \subset M$, and then set $U=M \backslash \Lambda$, we have by definition $N(M, U)=\operatorname{Perf}_{\Lambda} F(M)$. In particular, $\operatorname{Null}(M, \varnothing)=\operatorname{Perf} F(M)$.

Note as well in general $U_{1} \subset U_{2}$ implies $\operatorname{Null}\left(M, U_{2}\right) \subset \operatorname{Null}\left(M, U_{1}\right)$. 
Remark 5.12. As a consequence of our main results, we will deduce the nontrivial identity

$$
\operatorname{Null}(M, M) \simeq 0 \text {. }
$$

In other words, if a brane $L \in \operatorname{Perf} F(M)$ has empty singular support $\operatorname{ss}(L)=\varnothing$, then it itself is trivial $L \simeq 0$.

Definition 5.13. (1) We define the $\mathrm{st}_{k}$-valued presheaf $\mathcal{F}_{M}^{\mathrm{pre}}$ on the conic topology of $M$ by the assignment

$$
\mathcal{F}_{M}^{\mathrm{pre}}(U)=\operatorname{Perf} F(M) / \operatorname{Null}(M, U)
$$

for conic open subsets $U \subset M$.

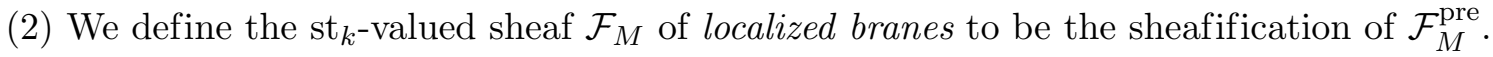

Remark 5.14. In order to obtain the essential gluing property of a sheaf, it is important that we follow Step (2) of the above definition and sheafify the naive quotient $\mathcal{F}_{M}^{\text {pre }}$. To see this explicitly, we recommend the reader continue on and consult Example 5.18 below where we illustrate this in the easiest possible situation.

For conic open subsets $U \subset M$, we have the canonical localization morphism

$$
\operatorname{Loc}_{U}: \operatorname{Perf} F(M) \longrightarrow \mathcal{F}_{M}^{\text {pre }}(U) \longrightarrow \mathcal{F}_{M}(U)
$$

Lemma 5.15. To each conic open subset $U \subset M$, and localized brane $\mathcal{L} \in \mathcal{F}_{M}(U)$, there is a unique conic closed subvariety $\operatorname{ss}_{U}(\mathcal{L}) \subset U$ called the localized singular support characterized by the properties:

(1) For conic open subsets $V \subset U \subset M$, we have compatibility with restriction

$$
\operatorname{ss}_{V}\left(\left.\mathcal{L}\right|_{V}\right)=\operatorname{ss}_{U}(\mathcal{L}) \cap V .
$$

(2) For a brane $L \in \operatorname{Perf} F(M)$, we have compatibility with global singular support

$$
\operatorname{ss}_{U}\left(\operatorname{Loc}_{U}(L)\right)=\operatorname{ss}(L) \cap U .
$$

Proof. The assertion is evident for sections of the presheaf $\mathcal{F}_{M}^{\text {pre }}$. Since conic closed subvarieties form a sheaf, the assertion follows for sections of the sheaf $\mathcal{F}_{M}$.

\subsection{Case of Weinstein cells}

Let $(N, \theta)$ be a Weinstein cell.

Lemma 5.16. If $L \in \operatorname{Perf} F(N)$ has empty singular support $\operatorname{ss}(L)=\varnothing$, then it itself is trivial $L \simeq 0$. In other words, we have $\operatorname{Null}(N, N) \simeq 0$.

Proof. By assumption, the unique zero $p \in N$ of the Liouville form lies in the null locus $n(L) \subset N$. Hence by definition, there exists a conic open set $U \subset N$ containing $p$ such that for any test brane $P \in F(N)$ with $P \subset U$, we have $\operatorname{hom}_{\operatorname{Perf} F(M)}(L, P) \simeq 0$. But $N$ itself is the unique conic open set $U \subset N$ containing $p$. Thus $\operatorname{hom}_{\operatorname{Perf} F(M)}(L, P) \simeq 0$ for any $P \in F(M)$, and hence $L \simeq 0$.

Proposition 5.17. Global localization is an equivalence

$$
\operatorname{Loc}_{N}: \operatorname{Perf} F(N) \stackrel{\sim}{\longrightarrow} \mathcal{F}_{N}(N) .
$$


Proof. Note that $N$ itself is the unique conic open set containing the unique zero $p \in N$ of the Liouville form. Hence any cover of $N$ by conic open sets must contain $N$ itself as a constituent. Thus the canonical map is an equivalence

$$
\mathcal{F}_{N}^{\text {pre }}(N) \stackrel{\sim}{\longrightarrow} \mathcal{F}_{N}(N)
$$

Finally, by the previous lemma $\operatorname{Null}(N, N) \simeq 0$, hence the canonical map is an equivalence

$$
\operatorname{Perf} F(N) \stackrel{\sim}{\longrightarrow} \mathcal{F}_{N}^{\text {pre }}(N) .
$$

Example 5.18. Returning to Definition 5.13, let us see the impact of sheafifying via the easiest possible example. Consider the two-dimensional Weinstein cell $M=\mathbb{C}$ with standard Liouville form $\theta$ and projectivization $M^{\infty} \simeq S^{1}$. Its core is the single point $K=\{0\} \subset \mathbb{C}$, and its ether is the complement $E=\mathbb{C}^{*} \subset \mathbb{C}$.

On the one hand, one can check that $\operatorname{Null}\left(\mathbb{C}, \mathbb{C}^{*}\right) \simeq 0$, and hence $\mathcal{F}_{M}^{\text {pre }}\left(\mathbb{C}^{*}\right) \simeq \operatorname{Perf} F(\mathbb{C})$. On the other hand, one can check that $\mathcal{F}_{M}\left(\mathbb{C}^{*}\right) \simeq \prod_{x \in S^{1}}$ Perf $k$, where the product is taken in st ${ }_{k}$. The image of the canonical morphism

$$
\operatorname{Perf} F(\mathbb{C}) \simeq \mathcal{F}_{M}^{\text {pre }}\left(\mathbb{C}^{*}\right) \longrightarrow \mathcal{F}_{M}\left(\mathbb{C}^{*}\right) \simeq \prod_{x \in S^{1}} \operatorname{Perf} k
$$

consists of sequences of objects whose underlying object (when we forget the labeling by points of $S^{1}$ ) is of the form $V \oplus V[1] \in$ Perf $k$. Informally speaking, the canonical morphism remembers the "ends" of branes near infinity, and any exact Lagrangian curve in $\mathbb{C}$ will have an even number of ends at infinity. More concretely, a brane $L \in \mathcal{F}_{M}^{\text {pre }}\left(\mathbb{C}^{*}\right) \simeq \operatorname{Perf} F(\mathbb{C})$ supported along the real line $\mathbb{R} \subset \mathbb{C}$, equipped with a rank one local system, will have endomorphisms $k$, but its image in $\mathcal{F}_{M}\left(\mathbb{C}^{*}\right) \simeq \prod_{x \in S^{1}}$ Perf $k$ will have endomorphisms $k \oplus k$.

Since $\mathcal{F}_{M}$ is a sheaf, we see that $\mathcal{F}_{M}^{\text {pre }}$ is not a sheaf, and hence it was important that we sheafified it.

\subsection{Compatibility with recollement}

Now assume the recollement pattern of diagrams (1.1) and (1.2) of the introduction as sketched in the preceding section.

Let $(M, \theta)$ be a Weinstein manifold.

Let $C \subset M$ be a closed coisotropic cell and consider the Hamiltonian reduction diagram

$$
N \ll \stackrel{q}{\longleftarrow} C \stackrel{i}{\longrightarrow} M,
$$

where $i$ is the inclusion of the coisotropic cell, and $q$ is the quotient by the integrable isotropic foliation determined by $i$.

Consider as well the complementary open

$$
j: M^{\circ}=M \backslash C \longleftrightarrow M .
$$

Proposition 5.19. Let $U^{\circ} \subset M^{\circ} \subset M$ be a conic open subset.

Then the recollement functors restrict to a diagram of adjunctions

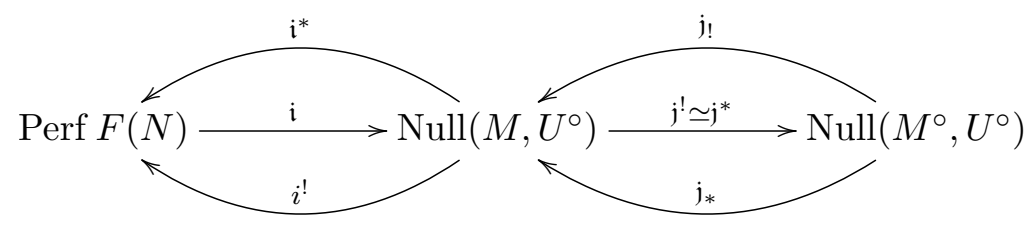


Proof. To avoid possible confusion, given a conic subset $A \subset M^{\circ} \subset M$, we will write $A_{M}^{\infty}$ and $A_{M^{\circ}}^{\infty}$ for its projectivizations as a subset of $M$ and $M^{\circ}$ respectively. Sinilarly, given a closed subset $A \subset M^{\circ} \subset M$, we will write $\partial_{M^{\infty}}^{\infty}$ and $\partial_{M^{\circ}}^{\infty} A$ for its boundaries at infinity as a subset of $M$ and $M^{\circ}$ respectively.

To see that the functor $i$ lands in $\operatorname{Null}\left(M, U^{\circ}\right)$, note first that $U^{\circ} \cap C=\varnothing$ and $\left(U^{\circ}\right)_{M}^{\infty} \cap C^{\infty}=$ $\varnothing$. Then any test brane $P \in F(M)$ with $P \subset U^{\circ}$ and $\partial_{M}^{\infty} P \subset\left(U^{\circ}\right)_{M}^{\infty}$ will not intersect a brane of the form $\mathfrak{i}(L) \in F(M)$, for any $L \in F(N)$.

From here, it suffices to see that the functor $j^{*}$ takes $\operatorname{Null}\left(M, U^{\circ}\right)$ to $\operatorname{Null}\left(M^{\circ}, U^{\circ}\right)$. Fix a brane $L \in \operatorname{Null}\left(M, U^{\circ}\right)$, and any point $x \in U^{\circ}$. We must confirm that $x \in n\left(\mathfrak{j}^{*}(L)\right)$.

Fix a conic open set $V \subset U^{\circ}$ containing $x$ that exhibits $x \in n(L)$. We will show that $V$ regarded as a subset of $M^{\circ}$ also exhibits $x \in n\left(\mathfrak{j}^{*}(L)\right)$.

Fix a test brane $P \in F\left(M^{\circ}\right)$ with $P \subset V$ and $\partial_{M^{\circ}}^{\infty} V \subset V_{M^{\circ}}^{\infty}$. Then we seek to show that

$$
\operatorname{hom}_{\operatorname{Perf} F\left(M^{\circ}\right)}\left(\mathfrak{j}^{*}(L), P\right) \simeq 0 .
$$

By adjunction, this is the same as to show that

$$
\operatorname{hom}_{P \operatorname{erf} F(M)}\left(L, \mathfrak{j}_{*}(P)\right) \simeq 0 \text {. }
$$

By construction, we have $\mathfrak{j}_{*}(P) \subset V$. After the small perturbation required to compute the above morphisms, we also have $\partial_{M}^{\infty}\left(\mathfrak{j}_{*}(P)\right) \subset V_{M}^{\infty}$. Thus since $V$ exhibits $x \in n(L)$, the above morphisms vanish, and hence $V$ also exhibits $x \in n\left(\mathfrak{j}^{*}(L)\right)$.

Corollary 5.20. Restriction induces a canonical equivalence

$$
\mathfrak{j}^{!} \simeq \mathrm{j}^{*}:\left.\mathcal{F}_{M}\right|_{M^{\circ}} \stackrel{\sim}{\longrightarrow} \mathcal{F}_{M^{\circ}} .
$$

Proof. It suffices to show the analogous statement for presheaves

$$
\left.\mathcal{F}_{M}^{\text {pre }}\right|_{M^{\circ}} \stackrel{\sim}{\longrightarrow} \mathcal{F}_{M^{\circ}}^{\text {pre }}
$$

In other words, for conic open subsets $U^{\circ} \subset M^{\circ}$, it suffices to show that the restriction descends to compatible equivalences

$$
\operatorname{Perf} F(M) / \operatorname{Null}\left(M, U^{\circ}\right) \stackrel{\sim}{\longrightarrow} \operatorname{Perf} F\left(M^{\circ}\right) / \operatorname{Null}\left(M^{\circ}, U^{\circ}\right) .
$$

This follows immediately from the recollement compatibility of Proposition 5.19.

Proposition 5.21. Let $U \subset M$ be a conic open subset containing $C$, and let $U^{\circ} \subset M^{\circ}$ denote the conic open subset $U^{\circ}=U \cap M^{\circ}$.

Then the recollement functors restrict to a diagram of equivalences

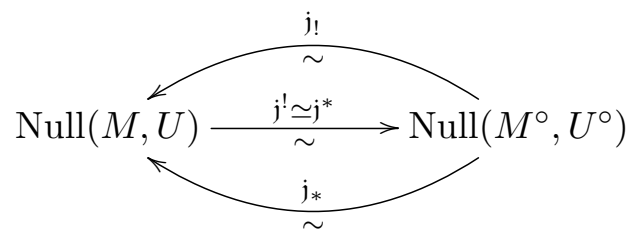

Proof. It is evident that $\mathfrak{j}_{!}, \mathfrak{j}_{*}$ take $\operatorname{Null}\left(M^{\circ}, U^{\circ}\right)$ to $\operatorname{Null}(M, U)$, and also that $\operatorname{Null}(M, U) \cap$ $\mathfrak{i}(\operatorname{Perf} F(M))=0$. 
Corollary 5.22. Let $U \subset M$ be a conic open subset containing $C$, and let $U^{\circ} \subset M^{\circ}$ denote the conic open subset $U^{\circ}=U \cap M^{\circ}$.

Then the recollement functors induce a diagram of adjunctions

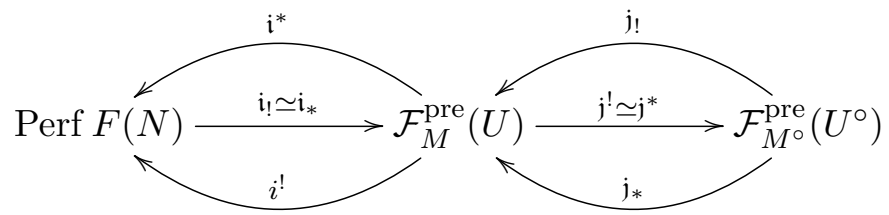

Consequently, we have an equivalence

$$
\mathcal{F}_{M}^{\text {pre }}(U) \simeq \operatorname{Mod}_{T}\left(\mathcal{F}_{M^{\circ}}^{\text {pre }}\left(U^{\circ}\right) \oplus \operatorname{Perf} F(N)\right),
$$

where $T=R L \in \operatorname{End}\left(\mathcal{F}_{M^{\circ}}^{\mathrm{pre}}(U) \oplus \operatorname{Perf} F(N)\right)$ is the monad of the adjunction

$$
L=\mathfrak{j}_{!} \oplus \mathfrak{i}_{!}: \mathcal{F}_{M^{\circ}}^{\text {pre }}\left(U^{\circ}\right) \oplus \operatorname{Perf} F(N) \longleftrightarrow \mathcal{F}_{M}^{\text {pre }}(U): R=\mathfrak{j}^{!} \oplus \mathfrak{i}^{!} .
$$

Proof. The first part follows immediately from the recollement compatibility of Proposition 5.21. The second part is an immediate application of the Barr-Beck theorem.

Remark 5.23. While the abstract monadic language is convenient, little of the sophisticated theory it represents is needed. More simply, we can say that $\mathcal{F}_{M}^{\mathrm{pre}}(U)$ is equivalent to the $\infty$-category of triples $L^{\circ} \in \mathcal{F}_{M^{\circ}}^{\mathrm{pre}}\left(U^{\circ}\right), L_{N} \in \operatorname{Perf} F(N)$ and a morphism

$$
r \in \operatorname{hom}_{\operatorname{Perf} F(N)}\left(\mathfrak{i}^{!} \mathfrak{j}_{!} L^{\circ}, L_{N}\right) .
$$

\subsection{Global sections}

Continue to assume the recollement pattern of diagrams (1.1) and (1.2) of the introduction.

Let $(M, \theta)$ be a Weinstein manifold.

Let $C \subset M$ be a closed coisotropic cell and consider the Hamiltonian reduction diagram

$$
N \stackrel{q}{\ll} C \stackrel{i}{\longrightarrow} M,
$$

where $i$ is the inclusion of the coisotropic cell, and $q$ is the quotient by the integrable isotropic foliation determined by $i$.

Consider as well the complementary open

$$
j: M^{\circ}=M \backslash C \smile \longrightarrow M .
$$

To find a natural context for Corollaries 5.20 and 5.22, let us consider the conic quotient

$$
\pi: M \longrightarrow M^{\sim}=M^{\circ} \cup *,
$$

where we collapse $C \subset M$ to a point denoted by $*$. Observe that the inverse-image under $\pi$ provides an equivalence from the category of conic open sets $U^{\sim} \subset M^{\sim}$ to the category of conic open sets $U \subset M$ such that $U \cap C$ is either all of $C$ or empty.

Let us introduce the pushforward presheaves $\pi_{*} \mathcal{F}_{M}^{\text {pre }}$ and $\pi_{*} j_{*} \mathcal{F}_{M^{\circ}}^{\text {pre }}$, and denote by $\operatorname{Perf} F(N)_{*}$ the skyscraper sheaf with fiber Perf $F(N)$ supported at $*$. Then we can reformulate Corollaries 5.20 and 5.22 as a diagram of adjunctions of presheaves

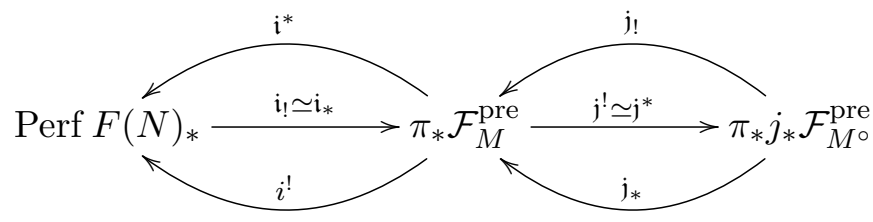


Consequently, we have an equivalence

$$
\pi_{*} \mathcal{F}_{M}^{\text {pre }} \simeq \operatorname{Mod}_{T}\left(\pi_{*} j_{*} \mathcal{F}_{M^{\circ}}^{\text {pre }} \oplus \operatorname{Perf} F(N)_{*}\right),
$$

where $T=R L \in \operatorname{End}\left(\pi_{*} j_{*} \mathcal{F}_{M^{\circ}}^{\text {pre }} \oplus \operatorname{Perf} F(N)_{*}\right)$ is the monad of the adjunction

$$
L=\mathfrak{j}_{!} \oplus \mathfrak{i}_{!}: \pi_{*} j_{*} \mathcal{F}_{M^{\circ}}^{\text {pre }} \oplus \operatorname{Perf} F(N)_{*} \rightleftarrows \pi_{*} \mathcal{F}_{M}^{\text {pre }}: R=\mathfrak{j} ! \oplus \mathfrak{i} !
$$

In concrete terms, to any conic open set $U^{\sim} \subset M^{\sim}$, we have that $\pi_{*} \mathcal{F}_{M}^{\text {pre }}\left(U^{\sim}\right)$ is equivalent to the $\infty$-category of triples $L^{\circ} \in \mathcal{F}_{M^{\circ}}^{\text {pre }}\left(U^{\circ}\right)$ where $U^{\circ}=U^{\sim} \cap M^{\circ}, L_{N} \in \operatorname{Perf} F(N)$ nonzero only if $* \in U^{\sim}$, and a morphism

$$
r \in \operatorname{hom}_{\operatorname{Perf} F(N)}\left(\mathfrak{i}^{!} \mathfrak{j} ! L^{\circ}, L_{N}\right) .
$$

Now we will check that the above description is compatible with sheafification.

Lemma 5.24. The canonical morphism is an equivalence of sheaves

$$
\left(\pi_{*} \mathcal{F}_{M}^{\mathrm{pre}}\right)^{+} \stackrel{\sim}{\longrightarrow} \pi_{*} \mathcal{F}_{M}
$$

Proof. This is evident over the open set $M^{\circ} \subset M^{\sim}$. It suffices to check that the canonical morphism induces an equivalence on stalks at $*$. This follows from the further observation that any conic open set $U \subset M$ containing the zero $p \in \pi^{-1}(*)=C$ in fact contains all of $C$.

Theorem 5.25. The pushforward $\pi_{*} \mathcal{F}_{M}$ admits the canonical description

$$
\pi_{*} \mathcal{F}_{M} \simeq \operatorname{Mod}_{T}\left(\pi_{*} j_{*} \mathcal{F}_{M^{\circ}} \oplus \operatorname{Perf} F(N)_{*}\right),
$$

where $T=R L \in \operatorname{End}\left(\pi_{*} j_{*} \mathcal{F}_{M^{\circ}} \oplus \operatorname{Perf} F(N)_{*}\right)$ is the monad of the adjunction

$$
L=\mathfrak{j}_{!} \oplus \mathfrak{i}_{!}: \pi_{*} j_{*} \mathcal{F}_{M^{\circ}}^{\text {pre }} \oplus \operatorname{Perf} F(N)_{*} \longleftrightarrow \mathcal{F}_{M}: R=\mathfrak{j}^{!} \oplus \mathfrak{i}^{!} .
$$

Proof. By our previous results reformulated above, the sheafification $\left(\pi_{*} \mathcal{F}_{M}^{\text {pre }}\right)^{+}$clearly admits the asserted description. Thus by the previous lemma, the pushforward $\pi_{*} \mathcal{F}_{M}$ does as well.

Corollary 5.26. Global localization is an equivalence

$$
\operatorname{Loc}_{M}: \operatorname{Perf} F(M) \stackrel{\sim}{\longrightarrow} \mathcal{F}_{M}(M)
$$

Proof. Note that $\pi_{*} \mathcal{F}_{M}\left(M^{\sim}\right) \simeq \mathcal{F}_{M}(M)$ and $\pi_{*} j_{*} \mathcal{F}_{M^{\circ}}\left(M^{\sim}\right) \simeq \mathcal{F}_{M^{\circ}}\left(M^{\circ}\right)$. By induction, global localization is an equivalence on the open Weinstein manifold

$$
\operatorname{Loc}_{M^{\circ}}: \operatorname{Perf} F\left(M^{\circ}\right) \stackrel{\sim}{\longrightarrow} \mathcal{F}_{M^{\circ}}\left(M^{\circ}\right)
$$

Hence by Theorem 5.25, we have an equivalence on global sections

$$
\mathcal{F}_{M}(M) \simeq \operatorname{Mod}_{T}\left(\operatorname{Perf} F\left(M^{\circ}\right) \oplus \operatorname{Perf} F(N)\right)
$$

where $T=R L \in \operatorname{End}\left(\operatorname{Perf} F\left(M^{\circ}\right) \oplus \operatorname{Perf} F(N)\right)$ is the monad of the adjunction

$$
L=\mathfrak{j}_{!} \oplus \mathfrak{i}_{!}: \operatorname{Perf} F\left(M^{\circ}\right) \oplus \operatorname{Perf} F(N) \rightleftarrows \mathcal{F}_{M}(M): R=\mathfrak{j}^{!} \oplus \mathfrak{i} !
$$

Comparison with the similar monadic description of Perf $F(M)$ yields the theorem. 
Corollary 5.27. For $L \in \operatorname{Perf} F(M)$, if $\operatorname{ss}(L)=\varnothing$, then $L \simeq 0$.

Proof. The localization of $L$ is a null brane for any conic open set.

Remark 5.28. We will not need the following discussion but include it to help further orient the interested reader.

One might ask whether a recollement description similar to that of Theorem 5.25 might exist for the sheaf $\mathcal{F}_{M}$ itself rather than its pushforward $\pi_{*} \mathcal{F}_{M}$. The immediate answer is negative since the key functors $\mathfrak{j}_{!}, \mathfrak{j}_{*}$ are not local. But one need not pass all the way to the quotient $M \rightarrow M^{\sim}$ induced by the collapse $C \rightarrow *$. Rather it is possible to pass to the intermediate quotient $M \rightarrow{ }^{\prime} M^{\sim}$ induced by the natural collapse $C \rightarrow N$. More broadly, the sheaf which admits a natural recollement pattern is the pushforward of $\mathcal{F}_{M}$ along the quotient of $M$ where each coisotropic cell is collapsed to its corresponding Weinstein cell.

In another direction, one might also ask which aspects of the recollement pattern can be lifted to the sheaf $\mathcal{F}_{M}$ itself. First, we can consider the full subsheaf $i_{!} q^{*} \mathcal{F}_{N} \subset \mathcal{F}_{M}$ generated by objects of the form $\mathfrak{i}(L) \in \operatorname{Perf} F(M)$, for objects $L \in \operatorname{Perf} F(N)$. There is a canonical equivalence on global sections

$$
\operatorname{Perf} F(N) \simeq \mathcal{F}_{N}(N) \stackrel{\sim}{\longrightarrow} i_{!} q^{*} \mathcal{F}_{N}(M)
$$

Second, we have seen that there is a canonical morphism $\mathcal{F}_{M} \rightarrow j_{*} \mathcal{F}_{M}$ 。 that induces an equivalence

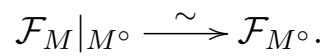

Unfortunately, as mentioned above, there are no evident adjoint maps of sheaves. The following related construction provides a partial solution. For $\dagger=$ ! or $*$, we can consider the full subsheaf $\mathcal{F}_{M^{\circ} \dagger} \subset \mathcal{F}_{M}$ generated by objects of the form $\mathfrak{j}_{\dagger} L \in \operatorname{Perf} F(M)$, for objects $L \in \operatorname{Perf} F\left(M^{\circ}\right)$. (We caution the reader that $\mathcal{F}_{M^{\circ} \dagger}$ is not the same as the pushforward $j_{\dagger} \mathcal{F}_{M}$.) Then restriction induces a canonical equivalence

$$
\left.\mathcal{F}_{M \dagger}\right|_{M^{\circ}} \stackrel{\sim}{\longrightarrow} \mathcal{F}_{M^{\circ}}
$$

Furthermore, it also induces a canonical equivalence on global sections

$$
\mathcal{F}_{M \dagger}(M) \stackrel{\sim}{\longrightarrow} \mathcal{F}_{M^{\circ}}\left(M^{\circ}\right)
$$

\subsection{Prescribed support}

Continue to assume the recollement pattern of diagrams (1.1) and (1.2) of the introduction.

Now let us introduce a characteristic cone $\Lambda \subset M$ so that we have a marked Weinstein manifold $(M, \theta, \Lambda)$. Recall by Lemma 5.15, we have the notion of singular support for localized branes.

Definition 5.29. We define the full subsheaf $\mathcal{F}_{\Lambda} \subset \mathcal{F}_{M}$ to consist of those localized branes $\mathcal{L} \subset \mathcal{F}_{M}(U)$ such that

$$
\operatorname{ss}_{U}(\mathcal{L}) \subset \Lambda \cap U
$$

for any conic open set $U \subset M$.

We now have the following assertion from the introduction. 
Theorem 5.30. Assume the recollement pattern of diagrams (1.1) and (1.2) of the introduction. Let $(M, \theta, \Lambda)$ be a marked Weinstein manifold.

The st ${ }_{k}$-valued sheaf $\mathcal{F}_{\Lambda}$ on the conic topology of $M$ has the following properties:

(1) The support of $\mathcal{F}_{\Lambda}$ is the characteristic cone $\Lambda \subset M$.

(2) The global sections of $\mathcal{F}_{\Lambda}$ are canonically equivalent to $\operatorname{Perf}_{\Lambda}(M)$.

(3) The restriction of $\mathcal{F}_{\Lambda}$ to an open Weinstein submanifold $M^{\circ} \subset M$ is canonically equivalent to the sheaf $\mathcal{F}_{\Lambda^{\circ}}$ constructed with respect to $\Lambda^{\circ}=\Lambda \cap M^{\circ}$.

(4) For each zero $p \in \mathfrak{c}$, the sections of $\mathcal{F}_{\Lambda}$ lying strictly above the unstable cell $C_{p} \subset M$ are canonically equivalent to $\operatorname{Perf}_{\Lambda_{p}}\left(M_{p}\right)$.

Example 5.31. Let us return to the setting of Example 5.18 and add a characteristic cone to the mix.

Recall the two-dimensional Weinstein cell $M=\mathbb{C}$ with standard Liouville form $\theta$ and projectivization $M^{\infty} \simeq S^{1}$. Its core is the single point $K=\{0\} \subset \mathbb{C}$, and its ether is the complement $E=\mathbb{C}^{*} \subset \mathbb{C}$. Any characteristic cone $\Lambda \subset \mathbb{C}$ will be the union of $K=\{0\}$ with finitely many rays. Let us denote by $\Lambda_{n} \subset \mathbb{C}$ the characteristic cone with $n$ rays, for $n=0,1,2, \ldots$.

Then as is well known, $\operatorname{Perf}_{\Lambda_{n}} F(\mathbb{C})$ is equivalent to finite-dimensional modules over the $A_{n-1}$-quiver (in particular, for $n=0$ and $n=1$, it is the zero category). This is also the stalk of the sheaf $\mathcal{F}_{\Lambda_{n}}$ at the point $0 \in \mathbb{C}$. Its stalk at other points $x \in \mathbb{C}$ is (not necessarily canonically) equivalent to Perf $k$ when $x \in \Lambda_{n}$, and the zero category otherwise.

\section{Acknowledgements}

I am indebted to D. Ben-Zvi, P. Seidel and E. Zaslow for the impact they have had on my thinking about symplectic and homotopical geometry. I am grateful to T. Perutz and D. Treumann for many stimulating discussions, both of a technical and philosophical nature. I am grateful to M. Abouzaid and D. Auroux for their patient explanations of foundational issues and related questions in mirror symmetry. I am also grateful to the anonymous referees for their thoughtful reading and generous investment in improving the paper. I would like to thank A. Preygel for sharing his perspective on ind-coherent sheaves. I am also pleased to acknowledge the motivating influence of a question asked by C. Teleman at ESI in Vienna in January 2007. Finally, I am grateful to the participants of the June 2011 MIT RTG Geometry retreat for their inspiring interest in this topic.

This work was supported by NSF grant DMS-0600909.

\section{References}

[1] Abouzaid M., A topological model for the Fukaya categories of plumbings, J. Differential Geom. 87 (2011), 1-80, arXiv:0904.1474.

[2] Abouzaid M., On the wrapped Fukaya category and based loops, J. Symplectic Geom. 10 (2012), 27-79, arXiv:0904.1474.

[3] Abouzaid M., Auroux D., Efimov A.I., Katzarkov L., Orlov D., Homological mirror symmetry for punctured spheres, J. Amer. Math. Soc. 26 (2013), 1051-1083, arXiv:1103.4322.

[4] Abouzaid M., Seidel P., An open string analogue of Viterbo functoriality, Geom. Topol. 14 (2010), 627-718, arXiv:0712.3177.

[5] Audin M., Lalonde F., Polterovich L., Symplectic rigidity: Lagrangian submanifolds, in Holomorphic Curves in Symplectic Geometry, Progr. Math., Vol. 117, Birkhäuser, Basel, 1994, 271-321.

[6] Auroux D., Fukaya categories and bordered Heegaard-Floer homology, in Proceedings of the International Congress of Mathematicians. Vol. II, Hindustan Book Agency, New Delhi, 2010, 917-941, arXiv:1003.2962. 
[7] Auroux D., Fukaya categories of symmetric products and bordered Heegaard-Floer homology, J. Gökova Geom. Topol. GGT 4 (2010), 1-54, arXiv:1001.4323.

[8] Beilinson A., Bernstein J., A proof of Jantzen conjectures, in I.M. Gel'fand Seminar, Adv. Soviet Math., Vol. 16, Amer. Math. Soc., Providence, RI, 1993, 1-50.

[9] Beilinson A.A., Coherent sheaves on $\mathbf{P}^{n}$ and problems in linear algebra, Funct. Anal. Appl. 12 (1978), $214-216$

[10] Ben-Zvi D., Francis J., Nadler D., Integral transforms and Drinfeld centers in derived algebraic geometry, J. Amer. Math. Soc. 23 (2010), 909-966, arXiv:0805.0157.

[11] Bierstone E., Milman P.D., Semianalytic and subanalytic sets, Inst. Hautes Études Sci. Publ. Math. (1988), $5-42$.

[12] Biran P., Lagrangian barriers and symplectic embeddings, Geom. Funct. Anal. 11 (2001), 407-464.

[13] Biran P., Lagrangian non-intersections, Geom. Funct. Anal. 16 (2006), 279-326, math.SG/0412110.

[14] Bourgeois F., Ekholm T., Eliashberg Y., Symplectic homology product via Legendrian surgery, Proc. Natl. Acad. Sci. USA 108 (2011), 8114-8121, arXiv:1012.4218.

[15] Bourgeois F., Ekholm T., Eliashberg Y., Effect of Legendrian surgery, Geom. Topol. 16 (2012), 301-389, arXiv:0911.0026.

[16] Braden T., Licata A., Proudfoot N., Webster B., Hypertoric category O , Adv. Math. 231 (2012), 1487-1545, arXiv:1010.2001.

[17] Cieliebak K., Handle attaching in symplectic homology and the chord conjecture, J. Eur. Math. Soc. 4 (2002), 115-142.

[18] Cieliebak K., Eliashberg Y., Symplectic geometry of Stein manifolds, 2006, unpublished.

[19] Eliashberg Y., Symplectic geometry of plurisubharmonic functions, in Gauge Theory and Symplectic Geometry (Montreal, PQ, 1995), NATO Adv. Sci. Inst. Ser. C Math. Phys. Sci., Vol. 488, Kluwer Acad. Publ., Dordrecht, 1997, 49-67.

[20] Eliashberg Y., Gromov M., Convex symplectic manifolds, in Several Complex Variables and Complex Geometry, Part 2 (Santa Cruz, CA, 1989), Proc. Sympos. Pure Math., Vol. 52, Amer. Math. Soc., Providence, RI, 1991, 135-162.

[21] Fukaya K., Oh Y.-G., Zero-loop open strings in the cotangent bundle and Morse homotopy, Asian J. Math. 1 (1997), 96-180.

[22] Fukaya K., Oh Y.-G., Ohta H., Ono K., Lagrangian intersection Floer theory - anomaly and obstruction, Kyoto Preprint, Math 00-17, 2000.

[23] Gordon I., Stafford J.T., Rational Cherednik algebras and Hilbert schemes, Adv. Math. 198 (2005), 222-274, math.RA/0407516.

[24] Gordon I., Stafford J.T., Rational Cherednik algebras and Hilbert schemes. II. Representations and sheaves, Duke Math. J. 132 (2006), 73-135, math.RT/0410293.

[25] Gotay M.J., On coisotropic imbeddings of presymplectic manifolds, Proc. Amer. Math. Soc. 84 (1982), 111-114.

[26] Kashiwara M., Rouquier R., Microlocalization of rational Cherednik algebras, Duke Math. J. 144 (2008), 525-573, arXiv:0705.1245.

[27] Kashiwara M., Schapira P., Sheaves on manifolds, Grundlehren der Mathematischen Wissenschaften, Vol. 292, Springer-Verlag, Berlin, 1994.

[28] Khovanov M., Seidel P., Quivers, Floer cohomology, and braid group actions, J. Amer. Math. Soc. 15 (2002), 203-271, math.QA/0006056.

[29] Kontsevich M., Symplectic geometry of homological algebra, 2009, available at http://www.ihes.fr/ $\sim \operatorname{maxim} /$ TEXTS/Symplectic_AT2009.pdf.

[30] Kostant B., On Whittaker vectors and representation theory, Invent. Math. 48 (1978), 101-184.

[31] Loday J.L., The diagonal of the Stasheff polytope, in Higher Structures in Geometry and Physics, Progr. Math., Vol. 287, Birkhäuser/Springer, New York, 2011, 269-292, arXiv:0710.0572.

[32] Losev I., Finite $W$-algebras, in Proceedings of the International Congress of Mathematicians. Vol. III, Hindustan Book Agency, New Delhi, 2010, 1281-1307, arXiv:1003.5811.

[33] Losev I., Quantized symplectic actions and W-algebras, J. Amer. Math. Soc. 23 (2010), 35-59, arXiv:0707.3108. 
[34] Lurie J., Higher algebra, available at http://www.math.harvard.edu/ lurie/.

[35] Lurie J., Higher topos theory, Annals of Mathematics Studies, Vol. 170, Princeton University Press, Princeton, NJ, 2009, math.CT/0608040.

[36] Lurie J., On the classification of topological field theories, in Current Developments in Mathematics, 2008, Int. Press, Somerville, MA, 2009, 129-280, arXiv:0905.0465.

[37] Manolescu C., Nilpotent slices, Hilbert schemes, and the Jones polynomial, Duke Math. J. 132 (2006), 311-369, math.SG/0411015.

[38] Markl M., Shnider S., Associahedra, cellular $W$-construction and products of $A_{\infty}$-algebras, Trans. Amer. Math. Soc. 358 (2006), 2353-2372, math.AT/0312277.

[39] Ma'u S., Gluing pseudoholomorphic quilted disks, arXiv:0909.3339.

[40] McGerty K., Nevins T., Derived equivalence for quantum symplectic resolutions, arXiv:1108.6267.

[41] Nadler D., Microlocal branes are constructible sheaves, Selecta Math. (N.S.) 15 (2009), 563-619, math.SG/0612399.

[42] Nadler D., Springer theory via the Hitchin fibration, Compos. Math. 147 (2011), 1635-1670, arXiv:0806.4566.

[43] Nadler D., Zaslow E., Constructible sheaves and the Fukaya category, J. Amer. Math. Soc. 22 (2009), 233-286, math.SG/0604379.

[44] Oh Y.-G., Unwrapped continuation invariance in Lagrangian Floer theory: energy and $C^{0}$ estimates, arXiv:0910.1131.

[45] Perutz T., Lagrangian matching invariants for fibred four-manifolds. I, Geom. Topol. 11 (2007), 759-828, math.SG/0606061.

[46] Perutz T., Lagrangian matching invariants for fibred four-manifolds. II, Geom. Topol. 12 (2008), 1461-1542, math.SG/0606062.

[47] Perutz T., A symplectic Gysin sequence, arXiv:0807.1863.

[48] Premet A., Special transverse slices and their enveloping algebras, Adv. Math. 170 (2002), 1-55.

[49] Saneblidze S., Umble R., A diagonal on the associahedra, math.AT/0011065.

[50] Saneblidze S., Umble R., Diagonals on the permutahedra, multiplihedra and associahedra, Homology Homotopy Appl. 6 (2004), 363-411, math.AT/0209109.

[51] Seidel P., More about vanishing cycles and mutation, in Symplectic Geometry and Mirror Symmetry (Seoul, 2000), World Sci. Publ., River Edge, NJ, 2001, 429-465, math.SG/0010032.

[52] Seidel P., Vanishing cycles and mutation, in European Congress of Mathematics, Vol. II (Barcelona, 2000), Progr. Math., Vol. 202, Birkhäuser, Basel, 2001, 65-85, math.SG/0007115.

[53] Seidel P., A long exact sequence for symplectic Floer cohomology, Topology 42 (2003), 1003-1063, math.SG/0105186.

[54] Seidel P., $A_{\infty}$-subalgebras and natural transformations, Homology, Homotopy Appl. 10 (2008), 83-114, math.KT/0701778.

[55] Seidel P., Fukaya categories and Picard-Lefschetz theory, Zurich Lectures in Advanced Mathematics, European Mathematical Society (EMS), Zürich, 2008, math.SG/0206155.

[56] Seidel P., Symplectic homology as Hochschild homology, in Algebraic Geometry - Seattle 2005. Part 1, Proc. Sympos. Pure Math., Vol. 80, Amer. Math. Soc., Providence, RI, 2009, 415-434, math.SG/0609037.

[57] Seidel P., Cotangent bundles and their relatives, morse Lectures, Princeton University, 2010, available at http://www-math.mit.edu/ seidel/talks/morse-lectures-1.pdf.

[58] Seidel P., Homological mirror symmetry for the genus two curve, J. Algebraic Geom. 20 (2011), 727-769, arXiv:0812.1171.

[59] Seidel P., Some speculations on pairs-of-pants decompositions and Fukaya categories, in Surveys in Differential Geometry. Vol. XVII, Surv. Differ. Geom., Vol. 17, Int. Press, Boston, MA, 2012, 411-425, arXiv:1004.0906.

[60] Seidel P., Smith I., A link invariant from the symplectic geometry of nilpotent slices, Duke Math. J. 134 (2006), 453-514, math.SG/0405089.

[61] Sheridan N., On the homological mirror symmetry conjecture for pairs of pants, J. Differential Geom. 89 (2011), 271-367, arXiv:1012.3238. 
[62] Sibilla N., Treumann D., Zaslow E., Ribbon graphs and mirror symmetry I, arXiv:1103.2462.

[63] Sikorav J.C., Some properties of holomorphic curves in almost complex manifolds, in Holomorphic curves in symplectic geometry, Progr. Math., Vol. 117, Birkhäuser, Basel, 1994, 165-189.

[64] Stasheff J.D., Homotopy associativity of H-spaces. I, Trans. Amer. Math. Soc. 108 (1963), 275-292.

[65] Stasheff J.D., Homotopy associativity of H-spaces. II, Trans. Amer. Math. Soc. 108 (1963), $293-312$.

[66] van den Dries L., Miller C., Geometric categories and o-minimal structures, Duke Math. J. 84 (1996), 497-540.

[67] Wehrheim K., Woodward C.T., Exact triangle for fibered Dehn twists, available at http://math. berkeley . edu/ katrin/.

[68] Wehrheim K., Woodward C., Pseudoholomorphic quilts, arXiv:0905.1369.

[69] Wehrheim K., Woodward C.T., Functoriality for Lagrangian correspondences in Floer theory, Quantum Topol. 1 (2010), 129-170, arXiv:0708.2851.

[70] Wehrheim K., Woodward C.T., Quilted Floer cohomology, Geom. Topol. 14 (2010), 833-902, arXiv:0905.1370.

[71] Wehrheim K., Woodward C.T., Floer cohomology and geometric composition of Lagrangian correspondences, Adv. Math. 230 (2012), 177-228, arXiv:0905.1368.

[72] Wehrheim K., Woodward C.T., Quilted Floer trajectories with constant components: corrigendum to the article "Quilted Floer cohomology", Geom. Topol. 16 (2012), 127-154, arXiv:1101.3770.

[73] Weinstein A., Lectures on symplectic manifolds, Regional Conference Series in Mathematics, Vol. 29, Amer. Math. Soc., Providence, R.I., 1977.

[74] Weinstein A., Neighborhood classification of isotropic embeddings, J. Differential Geom. 16 (1981), 125-128.

[75] Weinstein A., Contact surgery and symplectic handlebodies, Hokkaido Math. J. 20 (1991), 241-251. 